\title{
Projective Distribution of XQuery with Updates
}

\author{
Ying Zhang, Nan Tang, and Peter Boncz
}

\begin{abstract}
We investigate techniques to automatically decompose any XQuery query-including updating queries specified by the XQuery Update Facility (XQUF)—into subqueries, that can be executed near their data sources, i.e., function-shipping. The main challenge addressed here is to ensure that the decomposed queries properly respect XML node identity and preserve structural properties, when (parts of) XML nodes are sent over the network, effectively copying them. We start by precisely characterizing the conditions, under which pass-by-value parameter passing causes semantic differences between remote execution of an XQuery expression and its local execution. We then formulate a conservative strategy that effectively avoids decomposition in such cases. To broaden the possibilities of query distribution, we extend the pass-by-value semantics to a pass-by-fragment semantics, which keeps better track of node identities and structural properties. The pass-by-fragment semantics is subsequently refined to a pass-byprojection semantics by means of a novel runtime XML projection technique, which safely eliminates most semantic differences between the local and remote execution of an XQuery expression, and strongly reduces message sizes. Finally, we discuss how these techniques can be used for updating queries, both under the standard W3C XQUF specification, as well as under an extended semantics that allows to update remote documents. The proposed techniques are implemented in XRPC, a simple yet efficient XQuery extension that enables function-shipping by adding a Remote Procedure Call mechanism to XQuery. Experiments on MonetDB/ XQuery establish the performance potential of our XQuery decomposition techniques.
\end{abstract}

Index Terms-Distributed databases, XQuery decomposition.

\section{INTRODUCTION}

$I^{\mathrm{N}}$ this paper, we study ways to decompose any XQUERY query that consults multiple XML documents residing on multiple peers into subqueries that can be executed on those peers, i.e., function shipping. In principle, we do not want to restrict the form of these queries in any significant way: the full W3C recommended XQUERY language [3] including its XQUF extension [7] is the starting point of our decomposition. Our only requirement for peers to participate is running an XML database system (XDBMS) that complies with these W3C recommendations. The goal of this paper is to exploit the computational power of heterogeneous XML engines on the Web to jointly execute XQUERY and XQUF queries.

XQUERY already allows queries over distributed sources through its support for W3C standards, in particular, the ability to open any document on the Web through its fn:doc(URI) built-in function. However, the execution model implied by those W3C standards (e.g., HTTP) is data shipping: a full XML document is transported from a remote peer to the querying peer. This means that all query execution happens locally, i.e., at the query originator. It is well known that in many cases this is suboptimal. For instance, an aggregation query on a huge remote XML document that produces only a small result, incurs much less network cost when the aggregation is computed remotely (function shipping) than when the huge XML is shipped to the querying peer (data shipping).

- The authors are with the Centrum Wiskunde \& Informatica (CWI), PO Box 94079, NL-1090 GB Amsterdam, The Netherlands.

E-mail: \{Y.Zhang, N.Tang, P.Boncz\}@cwi.nl.

Manuscript received 15 May 2009; revised 15 Sept. 2009; accepted 14 Nov. 2009; published online 11 Apr. 2010.

Recommended for acceptance by Y. Ioannidis, D. Lee, and R. Ng.

For information on obtaining reprints of this article, please send e-mail to: tkde@computer.org, and reference IEEECS Log Number

TKDESI-2009-05-0430.

Digital Object Identifier no. 10.1109/TKDE.2010.62.

$1041-4347 / 10 / \$ 26.00 @ 2010$ IEEE
Decomposing queries to address multiple data sources is a well-studied optimization problem in relational [34], object-oriented [15], [20], and semistructured databases [30], [31]. While it is natural (and correct) to assume that many of the existing techniques can be carried over, the XML data model and the XQUERY language introduce a number of particular challenges not met elsewhere, that revolve around XML node identities and structural (rather than value-based) relationships between nodes. Previous work on distributed XML [6], [8], [32] only focused on a restricted subset of XQUERY queries, and did not address the problem of transparent query decomposition, such that these challenges did not arise.

Shipping XML messages. Without loss of generality, we view the subexpressions to be executed by remote peers as XQUERY functions, that may have parameters and produce a result. During remote function execution, the calling peer (e.g., query originator) will send a request message containing parameters to a remote peer, which executes the subexpression, and sends back a response message containing the result. To illustrate the challenges of distributing XQUERY, yet preserving XML node identity, consider a subexpression $f(\$ a, \$ b)$ with two parameters $\$ a$ and $\$ b$ of the type node(), that is executed remotely. Complications may arise, for instance, if the subexpression $f()$ tests structural XML relationships among its parameters, such as $" \$ a /$ parent::b is $\$ b "$. It therefore depends on the characteristics of the subexpressions $f()$, and on the way parameters are marshaled in and out of the network messages, to decide whether the distributed query will behave correctly. That is, whether the distributed query is identical to local execution (blindly copying all parameters into the message does not work in this example).

When XML nodes must be shipped over the network, this means that, unless one chooses to ship the entire XML document in order to preserve all structural relationships (which defeats the purpose of function shipping), 
pieces/snippets of the XML document must somehow be copied into the messages, changing the "holistic" structural properties and identities of nodes, which may affect the semantics of XQUERY execution on such shipped nodes. Naively, when shipping a node, one would ship its descendants (XML subtree), but other solutions are also possible, and will in fact be proposed in this paper (especially, the idea to use XML projection techniques). In particular, the runtime projection approach contributed in this paper tunes the shape of the shipped XML messages to the characteristics of the query, such that a minimal amount of data is shipped and those structural relationships that are actually needed are preserved.

Avoiding callbacks. One could consider a simple "callback" way of handling XQUERY distribution by not sending XML snippets at all, but just some (global) node identifiers. Each time when a peer needs to execute node-specific XQUERY (XPATH) expressions on such node identifiers, this alternative approach would communicate with the peer where the nodes originally came from, executing the nodespecific expressions on that peer and returning the results. While such an approach circumvents semantic problems, it has many drawbacks:

1. it basically gives up on the desire to move computation to more powerful peers;

2. it introduces additional network round-trips;

3. it makes all distributed queries-even read-only queries-stateful: a single query might consist of multiple (potentially many) network requests and the query processor on each peer must keep a session context open to guarantee repeatable reads consistency, which causes extra memory consumption and lock contention; and finally

4. there would be additional protocols needed to properly terminate such stateful distributed queries, adding extra protocol complexity, bookkeeping overhead, and network latencies.

In contrast, the techniques introduced here lead to flexible query distribution where subexpressions can be moved to the peer that can most efficiently process them. Typically, each peer is visited only once, thus network interactions are minimized and peers can handle the subqueries in a stateless manner. Additionally, one could also envision a network protocol that combines "callback" query processing with our techniques, something which might be interesting for handling distributed updating queries (because in the default XQUF semantics only locally stored documents can be updated, hence one would have to "callback" to the peer where a node originated from, to apply the update actions). However, given our target of Internet-wide P2P query processing with high network latencies, we decided against the "callback" approach in our own prototype construction, and fully focused on XQUERY execution by moving XML snippets and computation on them over the network. In this paper, we define and solve the problem this approach brings in preserving semantic correctness, and also demonstrate the efficiency of this approach.

XRPC. While our problem statement covers distributed XQUERY in general, this research stems from the particular context of the XRPC project [38], [39]. XRPC adds the concept

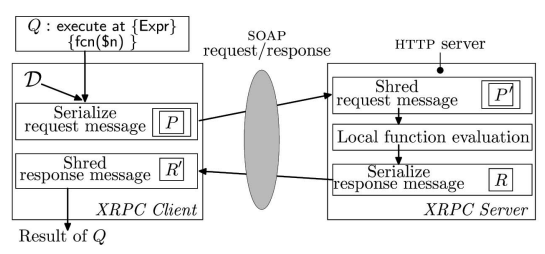

Fig. 1. XQUERY Remote Procedure Call under pass-by-value.

of Remote Procedure Call to XQUERY by introducing a new statement: execute at $\{$ ExprSingle\}\{Fun(Params)\}, where ExprSingle specifies the URI (a constant or a computed one) of the peer, on which Fun will be executed. As XQUERY is a compositional functional language, each query can be chopped up in arbitrary pieces. One can then view the pieces as functions connected together by function parameters and results. With XRPC, we have in principle ultimate flexibility in the way queries can be decomposed, as it allows each function to be executed on an arbitrary peer. An important feature on the network protocol level is bulk RPC that allows to handle multiple calls to the same function (with different parameters) in a single network interaction. Bulk RPC is exploited when a query contains a function call nested in an XQUERY for-loop, which in a naive implementation would lead to as many synchronous RPC network interactions as loop iterations. XRPC is implemented in MonetDB/XQuery [4], an open source XQUERY engine, which we use for experimental evaluation.

XRPC also supports a new URI scheme xrpc:// in the \$uri parameter of the built-in functions fn:put() and fn:doc(). Given a URL xrpc: $/ / \mathcal{P} / \mathcal{D}$, fn:put() stores the XML tree rooted at its $\$$ node parameter on the remote peer $\mathcal{P}$ as document $\mathcal{D}$, which possibly overwrites the existing $\mathcal{D}$. With fn:doc(), $\mathcal{D}$ could then be retrieved (over HTTP) from (the XRPC server on) peer $\mathcal{P}$. As we will see in Section 7, this extension enables supporting updates on remote documents identified by xrpc:// URIs.

Fig. 1 shows a query $Q$ that performs a single XRPC function call to $\mathrm{fcn}()$, with a single parameter (a node $\$ \mathrm{n}$ from some document $\mathcal{D}$ ). To make an XRPC call, the local peer formulates a SOAP request message, which contains a deep copy $P$ of the node $\$ n$. The Simple Object Access Protocol (SOAP) is an XML-based message format commonly used by web services [22]. XRPC follows the previously mentioned approach of copying the XML subtree of a node parameter, which implies a pass-by-value parameter passing strategy. The message is sent as a synchronous HTTP POST request. The remote peer runs an HTTP server, which parses the request message and constructs a separate XML fragment for each node parameter (in this example a single fragment $P^{\prime}$ ). The remote peer then evaluates the function and serializes the result into a response message (here, a deep copy of the result node, denoted $R$ ). Finally, the local peer parses the response message and constructs a separate XML fragment for each node-typed result (here $R^{\prime}$ ), which is the result of $Q$.

Problem statement. Our goal is to rewrite an XQUERY $Q$ that uses XML documents with xrpc:// URIs stored at remote peers, into an equivalent query $Q^{\prime}$ that uses XRPC calls to execute parts of the query (expressed as XQUERY functions) on those remote peers. For a query $Q, Q(\mathcal{D})$ denotes the result 
of evaluating $Q$ over a (possibly distributed) database $\mathcal{D}$. Two queries $Q$ and $Q^{\prime}$ are equivalent, if $Q(\mathcal{D})=Q^{\prime}(\mathcal{D})$ for any given database $\mathcal{D}$ (under the XQUERY deep-equal semantics).

We illustrate XQUERY decomposition as follows:

for $\$$ e in doc(“employees.xml”)//emp

where \$e/@dept = doc(“xrpc://example.org/depts.xml”)//dept/@name return $\$$ e

the URL xrpc://example.org/depts.xml implies that the remote peer example.org supports XRPC, so the predicates could be pushed as:

declare function fcn( $\$ n$ as xs:string) as xs:boolean

$\{\$ n=$ doc("depts.xml")//dept/@name $\}$;

for $\$$ e in doc("employees.xml")//emp

where execute at $\{$ "example.org" $\}\{$ fcn $(\$ e / @$ dept $)\}$ return $\$ e$

In this example, the parameter and return value of the function $\mathrm{fcn}()$ are of atomic types. In more complex cases, nodes may be involved, such that potential semantic differences due to pass-by-value should be considered (discussed in Section 2), which is our main challenge.

Contributions and road map. Section 2 identifies the semantic differences of remote XQUERY pass-by-value function evaluation with respect to standard, local function evaluation. Section 3 describes an XQUERY CORE-based query decomposition framework. This leads in Section 4 to a conservative XQUERY decomposition strategy that avoids semantic problems simply by refraining from decomposition in all problem cases. To make our rewrites more effective and robust against syntactic variation, we describe normalization and code motion rewrite strategies. As a second contribution, Section 5 extends the pass-by-value semantics with a new pass-by-fragment message format that conserves more structural relationships between nodes passed in a message and allows more predicates to be distributed. Section 6 introduces a new runtime XML projection technique, which we use to generate messages that conserve all needed structural relationships between transferred XML nodes, and thus allow even more freedom in query decomposition. As a runtime technique, it is able to prune XML data much more than previously described compile-time projections [2], [5], [18]. In Section 7.1, we discuss how updating queries can be handled, both in the normal XQUF semantics, as well as under an extension, in which we allow nonlocal documents to be updated. An evaluation of the performance benefits of our techniques is given in Section 8. We discuss related work in Section 9 and conclude in Section 10 with outlook on future work.

\section{Semantic Differences of Pass-By-Value}

There are well-defined semantic differences [38] between evaluating an XQUERY expression locally and executing it remotely under pass-by-value parameter passing. We discuss these differences with a query $Q_{1}$ in Table 1 . This query evaluates three functions: makenodes(), overlap(), and earlier().

Problem 1 (Nondownward XPath Steps). Reverse and horizontal XPATH axis navigation (e.g., parent, ancestor, preceding(-sibling), and following(-sibling)) from remote function parameters always produces empty results, as passby-value node serialization only includes the descendants of a node inside the message. Consider the following:
TABLE 1

Example Query $Q_{1}$

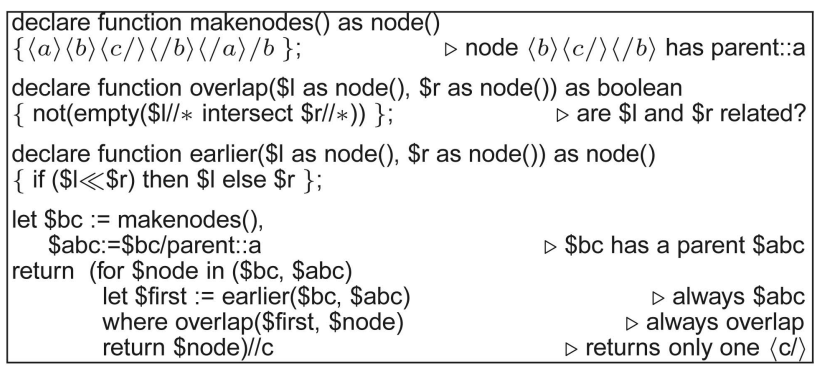

let $\$ b c:=$ execute at $\{$ "example.org" $\}$ makenodes ()$\}$, $\$ a b c:=\$ b c / p a r e n t:: a$

here, \$abc evaluates to the empty sequence, instead of the correct a-node $\langle a\rangle\langle b\rangle\langle c /\rangle\langle/ b\rangle\langle/ a\rangle$.

It is possible to evaluate downward XPATH steps on a sequence of remote nodes, but only if we are sure that these nodes are ordered and nonoverlapping (otherwise, the results of such XPATH steps will fail to respect node identity and order, as described below).

Problem 2 (Node Identity Comparisons). If a remote function returns a sequence with two identical nodes, or two identical nodes are passed as function parameters, pass-byvalue represents them as two different copies. This leads to the duplicate elimination problem described just above, and any node identity comparison will always yield false. For instance:

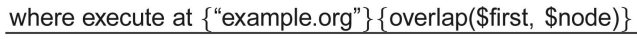

yields false, while the local query evaluation gives true.

Problem 3 (Document Order). The parameters of a function call on a remote peer are serialized into the message in parameter order, in separate XML fragments. Even if the parameter nodes are disjoint (making Problem 2 irrelevant), the relative order between these XML fragments may differ from their original order. Thus, interparameter node comparisons ("«", "》") may behave differently from the local semantics. Consider the usage of earlier() in $Q_{1}$ as:

$\underline{\text { let } \$ \text { first := execute at }\{\text { "example.org"\} \{earlier }(\$ b c, \$ a b c)\}}$

In both iterations, the variable \$first binds to a copy of $\$ b c$, instead of \$abc, although \$abc is the parent of $\$ b c$.

Another problem with document order, not revealed by this example, could occur when comparisons of nodes from different $X M L$ documents are executed on remote peers. The XQuery/ XPath Data Model (XDM) [12] defines that the relative order of nodes in different documents is implementation-dependent, but must be stable during the processing of the same query. Consider the query

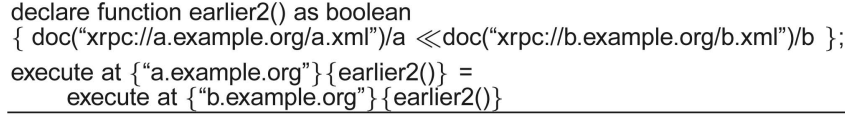

which, depending on how documents are ordered by the remote peers, could return true or false, ${ }^{1}$ while XDM requires it to

1. Even if the two calls to earlier2() were executed on the same remote peer, without any guarantees for consistency, the results could be different, since each call is a separate query on the remote peer. 
always return true. Note, however, that a query containing a single call to earlier2() may return either true or false, confirming XDM, then in such queries, earlier2() could be executed at a remote peer.

Problem 4 (Interaction Between Different Calls). Additional semantic differences can occur when XQUERY subexpressions (sequences) may contain nodes that were obtained as results from different remote function calls, and these function calls, directly or indirectly, accessed the same XML document on some peer. Node sequences can become intermixed by any XQUERY construct that accepts multiple inputs, namely: sequence construction, and the built-in functions union, except, and intersect. A special source of call-mixing is the return clause of a for-loop in which remote function evaluation is performed, because the return clause implicitly creates a sequence that concatenates the expression result of all loop iterations (each of which performed a semantically separate remote function call). The result of such "mixed-call expressions" is that nodes returned by different calls may in fact stem from the same document. However, node identity and ordering between nodes from different calls is not preserved, leading to semantic differences. For example, even if a downward XPATH step is applied on an input sequence containing nodes obtained from different remote calls, the result can have the wrong order (placing the results from the first call always before those of the second call) and will fail to properly eliminate duplicates:

( for \$node in (\$bc, \$abc)

let $\$$ first := execute at $\{$ "example.org" $\}$ earlier(\$node,\$abc) $\}$ return $\$$ node) $/ / c$

The above two XRPC calls produce nodes belonging to separate $X M L$ fragments. Under pass-by-value, evaluating //c produces two separate copies of c nodes, while in local execution the nodes returned from earlier() are from the same XML fragment, such that XPATH steps return a duplicate-free result.

Problem 5 (XQuery Built-In Functions). Various problems may occur when evaluating certain built-in functions remotely.

1. static-base-uri(), default-collation(), and currentdatetime(): depend on the static XQUERY context.

2. base-uri() and document-uri(): depend on the $d y$ namic context of node expressions.

3. root(): accesses the document root.

4. id() and idref(): return all nodes in a document with certain ID/IDREF values.

5. lang(): accesses the xml:lang attribute of the context node and its ancestors.

Class 1 of above built-in functions is handled by extending the XRPC message format with extra attributes such that the remote side can declare identical values for these context attributes. ${ }^{2}$ Class 2 is dealt with by adding these properties as attributes in the XRPC nodes (such as xrpc:element) that enclose serialised parameter/result nodes in the SOAP messages. Use of the fn:base-uri() and fn:document-uri() in XRPC is substituted by xrpc:base-uri() and xrpc:documenturi() wrappers that take these attributes into account when invoked on XRPC parameter nodes. As solutions for Class 1-2 are available, the main problem with built-in functions is posed

2. If static-base-uri() is not set, we ship the value $\mathrm{xrpc}: / / \mathcal{P} / \mathrm{doc} /$, so that fn:doc() calls with a relative document URI call back to the originating peer $\mathcal{P}$.
TABLE 2

Xcore Grammar Rules

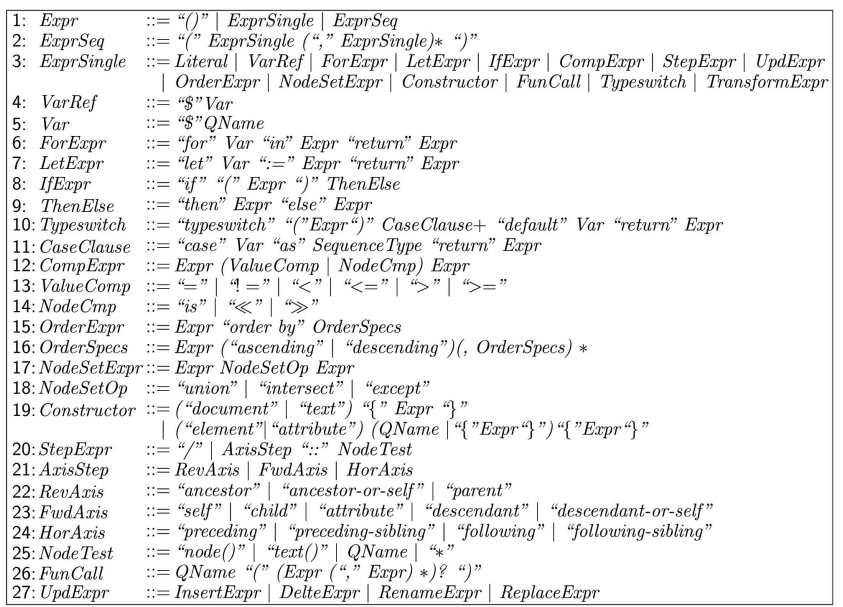

by Classes 3-5, which access nondescendants of parameter nodes, and thus cannot be supported with pass-by-value.

In the remainder, we present decomposition techniques and extensions to enhance the pass-by-value semantics, that solve the aforementioned problems.

\section{XQuery Core Rewrite Framework}

XQUERY CORE [10] (abbreviated XCORE) is a subset of XQUERY, in which all implicit operations are made explicit. We adopt a subset of XCORE expressions in Table 2, which is sufficient to capture XPATH 1.0 and XQUERY FLWOR expressions [10]. Additionally, we support all updating expressions (rule UpdExpr) and the transform expression (TransformExpr) as defined by XQUF. For reasons of space, we thus omit repeating grammar rules for those expressions. We use a representation of XPATH paths in our XCORE grammar that keeps consecutive steps together, rather than nesting each step in a separate for-loop (when allowed-the use of position() precludes this). Such an optimization is common in XQUERY engines, and is part of XQUERY normalization, further described in Section 4. Additionally, we define two new rules for the XRPC extension [38]:

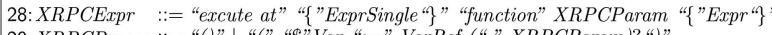
29:XRPCParam ::= "()" | "(" "\$"Var ":="VarRef ("," XRPCParam)?") "

Rule 28 identifies an xrpc:// URI in expression ExprSingle, and declares a new anonymous function that is to be executed remotely. It is noticeable that these grammar rules lack the expressive power to define recursive functions. This does not matter for XQUERY decomposition, as our decomposition strategies will not generate recursive functions. The syntax defined by rules 28 and 29 differs from the actual XRPC syntax (execute at ExprSingle\}\{Fun(Params)\}). The syntax used here is only for presentation purpose, to avoid the need to define all rules concerning declaration of userdefined functions. Thus, our simple XCORE rule without explicit user-defined function declarations allows to express all queries in a single ExprSingle, which in turn can be mapped to a query graph. This simplifies the formulation of analysis steps. 
TABLE 3

Example Query $Q_{2}$

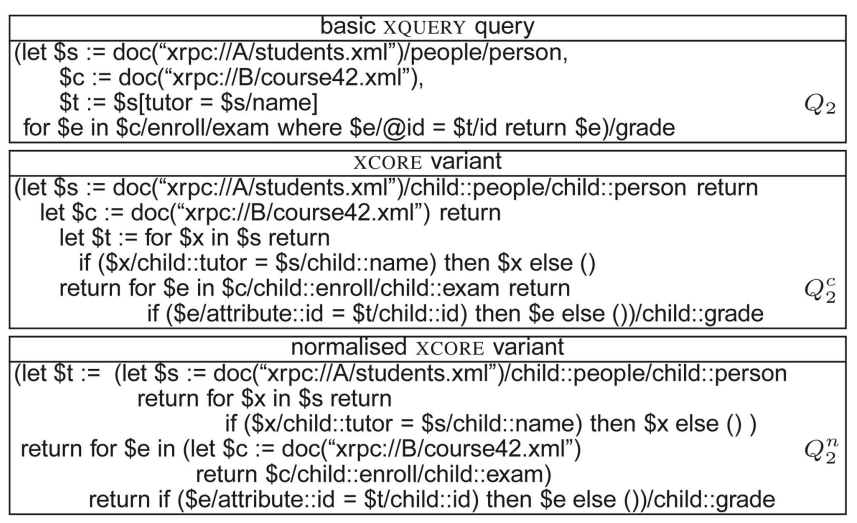

XCore dependency graph. We introduce a dependency graph ( $d$-graph) for an XCORE query. Consider the XQUERY query $Q_{2}$ in Table 3, which asks for the grade in course 42 of students having a tutor who is also a student, and its XCORE equivalence $Q_{2}^{c}$.

A dependency graph is a directed, ordered and connected graph $G$ with vertices $V(G)$ and edges $E(G)$. Each vertex $v$ is denoted as $v_{i}$ :rule[val], where $v_{i}$ is a unique vertex identifier, rule is the grammar rule represented by $v_{i}$, and val is an optional value indicating the right-hand-side of rule. There is a single root vertex without incoming edges. $E(G)$ consists of parse edges $E_{p}(G)$ and varref edges $E_{v}(G)$. Each parse edge is an ordered vertex pair $(u, v)$, where $u$ corresponds to a parsing rule $r_{u}$ that directly causes the use of another parsing rule $r_{v}$. A varrefedge is an ordered vertex pair $(w, x)$ denoting a variable usage. When a VarRef rule is used, an additional edge is created between the VarRef vertex and the Var vertex that defines the variable.

Example 3.1. Fig. 2 shows the $d$-graph of $Q_{2}^{n}$ in Table 3. Solid and dashed lines represent parse and varref edges, respectively. The variable binding in the first let expression corresponds to vertices $v_{2}, \ldots, v_{21}$, and vertices $v_{22}, \ldots, v_{39}$ depict its return clause. The edge $\left(v_{6}, v_{7}\right)$ is a parse edge. The edge $\left(v_{30}, v_{25}\right)$ is a varref edge, as the variable used by $v_{30}$ is a reference of variable $\$ c$ introduced by $v_{25}$. Thus, a d-graph is in essence a parse-tree with additional (dashed) edges to indicate variable usages.

We define three types of dependency relationships upon the reachability between two vertices $x, y$ in $V(G): 1) x$ "parse-depends on" $y$, denoted as $x \stackrel{p}{\sim} y$, if $y$ is reachable from $x$ via only parse edges; 2 ) $x$ "varref-depends on" $y$, denoted as $x \stackrel{v}{\sim} y$, if $y$ is reachable from $x$ via at least one varref edge; and 3) $x$ "depends on" $y$, denoted as $x \sim y$, if either $x \stackrel{p}{\sim} y$ or $x \stackrel{v}{\sim} y$ holds. The compositional nature of XQUERY means that $x \sim y$ concisely captures all semantic dependencies between subexpressions. Consider Fig. 2, $v_{15} \stackrel{p}{\sim} v_{16}$, since $\left(v_{15}, v_{16}\right)$ is a parse edge; $v_{15} \stackrel{v}{\sim} v_{11}$, as $v_{11}$ is reachable from $v_{15}$ via $\left(v_{15}, v_{16}\right),\left(v_{16}, v_{11}\right)$, and $\left(v_{16}, v_{11}\right)$ is a varref edge.

For a $d$-graph $G$ and a vertex $r_{s} \in V(G)$, we use the term subgraph to mean the vertex-induced subgraph of $r_{s}$,

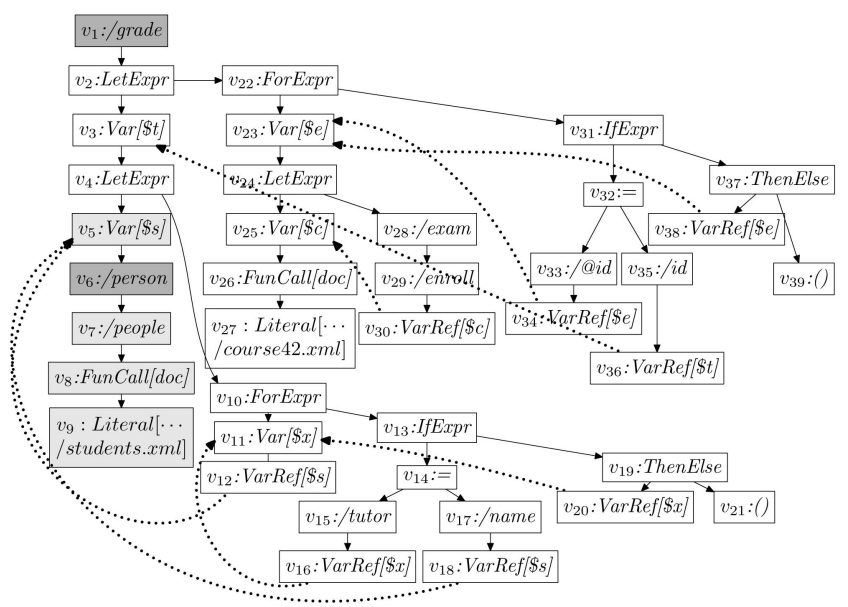

Fig. 2. $d$-graph of the normalized XCORE variant $Q_{2}^{n}$ in Table 3.

denoted $G_{r_{s}}$, including $r_{s}$ and all $u \in V(G)$ where $r_{s} \stackrel{p}{\sim} u$; $r_{s}$ is called the root of the subgraph. For instance, the subgraph rooted at vertex $v_{22}$ contains vertices $v_{22}, \ldots v_{39}$, but does not contain vertices $v_{3}, \ldots, v_{21}$. Throughout this paper, we use the terms (sub)graph and (sub)query interchangeably, as a (sub)query is represented by the induced subgraph rooted at some vertex.

XRPCExpr insertion. We can decide to evaluate a certain subgraph $G_{r_{s}}$ rooted at $r_{s}$ remotely over XRPC, by inserting a $v_{x}$ :XRPCExpr node above it. This may only be done if we can ensure that the result of the rewritten query is identical to the original query. Such an insertion means that a new function will be defined that contains $G_{r_{s}}$ as its body. In the main query graph, $G_{r_{s}}$ is replaced by a remote XRPC call to this function, which receives as parameters all variable references in $G_{r_{s}}$ that resolve to variable bindings outside $G_{r_{s}}$ :

1. Insert a vertex $v_{x}$ :XRPCExpr, a parse edge $\left(v_{x}, r_{s}\right)$, and replace each incoming edge $\left(v_{i n}, r_{s}\right)$ with a new edge $\left(v_{i n}, v_{x}\right)^{3}$

2. For each outgoing varref edge from vertex $v_{i} \in V\left(G_{r_{s}}\right)$ to $v_{j} \in V(G) \backslash V\left(G_{r_{s}}\right)$, where edge $\left(v_{i}, v_{j}\right) \in E_{v}(G)$ is a varref edge as $\left(v_{i}: \operatorname{VarRef}[\$ q n a m e], v_{j}: \operatorname{Var}[\right.$ \$qname $\left.]\right)$, we insert a new vertex $v_{k}$, a new parse edge $\left(v_{x}, v_{k}\right)$ and replace the varref edge $\left(v_{i}, v_{j}\right)$ by $\left(v_{i}, v_{k}\right)$ and $\left(v_{k}, v_{j}\right)$. Here, $v_{k}$ has the form $v_{k}$ :XRPCParam $[\$ p:=\$$ qname], which introduces a new variable $\$ p$ and binds it to \$qname in $v_{j}$.

3. If there are no outgoing edges as stated in step 2, we insert a vertex $v_{l}$ with the form $v_{l}$ :XRPCParam[()] (i.e., empty parameter), and a parse edge $\left(v_{x}, v_{l}\right)$.

Example 3.2. Consider the d-graph in Fig. 2. Suppose that the subgraph rooted at $v_{22}$ is identified for an XRPCExpr insertion (Fig. 3). First, insert vertex $v_{40}$ and replace edge $\left(v_{2}, v_{22}\right)$ by $\left(v_{2}, v_{40}\right)$ and $\left(v_{40}, v_{22}\right)$. For the outgoing varref edge $\left(v_{36}, v_{3}\right)$, vertex $v_{41}$ is inserted below $v_{40}$ and the varref edge is replaced by two new varref edges: $\left(v_{36}, v_{41}\right),\left(v_{41}, v_{3}\right)$.

3. Determined by the algorithms (Section 4.6) that compute the insertion points (i.e., determine if a vertex may be an $r_{s}$ ), $r_{s}$ is the only vertex in the subgraph $G_{r_{s}}$ that has incoming edges from vertex outside $G_{r_{s}}$. 


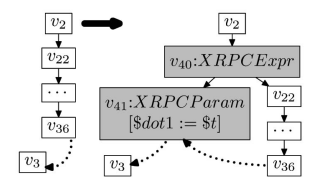

Fig. 3. XRPCExpr insertion.

\section{Conservative Decomposition}

In this and the next two sections, we first describe algorithms to decompose read-only XCORE queries. We delay the discussion of decomposing queries containing any UpdExpr and TransformExpr expressions until Section 7.

By-value insertion conditions. Given a $d$-graph $G$ and a subgraph $G_{r_{s}}$ of $G$ rooted at $r_{s}$, under the pass-by-value semantics, vertex $r_{s}$ is in the set $I(G)$ of valid decomposition points ( $d$-points), iff $r_{s}$ satisfies all of the following conditions:

1.

$$
\begin{gathered}
\nexists n \in V(G): \text { n.rule } \in\{\operatorname{RevAxis,HorAxis\} } \\
\wedge\left(\operatorname{useResult}\left(n, r_{s}\right) \vee \operatorname{useParam}\left(r_{s}, n\right)\right)
\end{gathered}
$$

2.

$$
\begin{aligned}
& \nexists n \in V(G): \text { n.rule } \in\{\text { NodeCmp, NodeSetExpr }\} \\
& \wedge\left(\left(r_{s} . \text { rule } \in\{\operatorname{NodeCmp}, \operatorname{NodeSetExpr}\} \wedge n\right.\right. \\
& \left.\left.\notin V\left(G_{r_{s}}\right)\right) \vee \operatorname{useResult}\left(n, r_{s}\right) \vee \operatorname{useParam}\left(r_{s}, n\right)\right) ;
\end{aligned}
$$

3.

$$
\begin{aligned}
& \nexists n \in V(G), \exists m \in V(G): n . r u l e=\text { AxisStep } \\
& \wedge m . r u l e \in\{\text { ForExpr, OrderExpr, ExprSeq, } \\
& \text { NodeSetExpr, AxisStep } \backslash\{\text { self, child, attribute }\}\} \\
& \wedge\left(( \text { useResult } ( n , m ) \wedge m \sim r _ { s } ) \vee \left(\text { useResult }\left(n, r_{s}\right)\right.\right. \\
& \left.\left.\wedge m \in V\left(G_{r_{s}}\right)\right) \vee\left(m \notin V\left(G_{r_{s}}\right) \wedge r_{s} \stackrel{p}{\sim} n \stackrel{v}{\sim} m\right)\right)
\end{aligned}
$$

4.

$$
\begin{aligned}
& \nexists n \in V(G): \text { n.rule }=\text { FunCall } \wedge \text { n.val } \in \\
& \{\mathrm{fn}: \operatorname{root}(), \mathrm{fn}: \operatorname{id}(), \mathrm{fn}: \operatorname{idref}(), \mathrm{fn}: \operatorname{lang}()\} \\
& \wedge\left(\operatorname{useResult}\left(n, r_{s}\right) \vee \operatorname{useParam}\left(r_{s}, n\right)\right) .
\end{aligned}
$$

where we impose these restrictions symmetrically both on expressions that use the result of the remote expression $r_{s}$, and on the way remote expressions (below $r_{s}$ ) use their shipped parameters:

$$
\begin{aligned}
& \operatorname{useResult}\left(n, r_{s}\right) \Leftrightarrow n \sim r_{s} \\
& \operatorname{useParam}\left(r_{s}, n\right) \Leftrightarrow n \in V\left(G_{r_{s}}\right), \exists v \in V(G) V\left(G_{r_{s}}\right): n \sim v
\end{aligned}
$$

Conditions 1 and 2 guard against using any node comparisons, and horizontal and reverse XPATH steps on shipped nodes, avoiding Problems 1-3 (Section 2). Condition 2 also disallows decomposing any node comparisons, when a query contains multiple such expressions, to avoid the problem with document order of nodes from different documents. Condition 3 avoids using downwards XPATH steps (per Condition 1) on shipped nodes from expressions that might be so-called "mixed-call sequences" (ForExpr, ExprSeq, NodeSetExpr), avoiding Problem 4. It also guards against sequences not in node order (ForExpr, OrderExpr) or with nodes that may be overlapping (the restrictions on NodeSetExpr and XPATH steps). This ensures that downwards XPATH steps can be used on shipped node sequences that are ordered and nonoverlapping. Condition 4 states that shipped nodes may not be used as parameters of the listed built-in functions (Problem 5).

Example 4.1. In the $d$-graph of example query $Q_{2}^{n}$ (Fig. 2), we mark in gray colors the $d$-points identified by the conservative decomposition strategy. The XPATH step / grade that is performed on the result of a for-loop, matches condition iii and causes all vertices that depend on $v_{10}$ and $v_{22}$ (the ForExprs) as well as all their descendants to be excluded from $I(G)$, leaving $v_{1}$ and the subgraphs rooted at $v_{5}$ as $d$-points.

Interesting decomposition points. While a $d$-point may be semantically valid, remote evaluation of the subquery below it might be senseless. Consider the $d$-point $v_{8}$, which contains only an fn:doc() function call in its subgraph. Executing this function remotely provides no performance gain, as it only demands the shipping of a whole document. Similarly, remote execution of expressions that do not involve any XML documents should be avoided. Therefore, we filter $d$-points by first annotating each vertex $v_{x} \in V(G)$ with the URI dependency set $D\left(v_{x}\right)$. Here, $D\left(v_{x}\right)$ represents the set of URIs that are used as parameters of fn:doc() in vertices that the vertex $v_{x}$ can reach via parse edges:

$$
\begin{aligned}
D\left(v_{x}\right)= & \left\{\text { uri }:: v_{y} \mid\left\{v_{y}, v_{z}\right\} \in E(G): \text { uri }=v_{z} \cdot \text { val } \wedge v_{x} \stackrel{p}{\sim} v_{y}\right. \\
& \wedge v_{y} \cdot \text { rule }=\text { FunApp } \wedge v_{y} \cdot v a l=" d o c " \wedge v_{z} \cdot \text { rule } \\
= & \text { Literal }\} .
\end{aligned}
$$

We tag each uri with the vertex $v_{y}$ where the document is opened, to be able to distinguish the use of the same document through multiple fn:doc() calls. This definition does not cover the case that the parameter of fn:doc() is an expression instead of a literal. In those cases, we use a wildcard symbol " $*$ " as uri. In this paper, the built-in function fn:collection() is treated as an fn:doc(*), and an element construction is assigned an artificial unique URI fn:doc $\left(v_{i}:: v_{i}\right)$.

One can use the URI dependency set to partition the $V(G)$ into equivalence classes, i.e., those vertices with the same URI dependency set belong to the same class. Using all vertices in an equivalence class, we can consider its induced subgraph in $G$, and try to handle it in a single XRPC subquery. Thus, we define interesting decomposition points ( $i$ points) $I^{\prime}(G)$ as those valid insertion points that 1 ) are a root vertex in their induced subgraph (if the root node happens to be a Var vertex, we consider its value expression instead as root), 2) contain at least one $f n: d o c()$ and 3) execute at least one XPATH step on the fn:doc() function:

$$
\begin{aligned}
I^{\prime}(G)= & \left\{v_{x} \mid v_{x} \in I(G): \nexists v_{y}: v_{y} \stackrel{p}{\sim} v_{x} \wedge D\left(v_{x}\right)=D\left(v_{y}\right)\right. \\
& \wedge \exists v_{z}: v_{x} \stackrel{p}{\sim} v_{z} \wedge v_{z} \cdot \text { rule }=\text { AxisStep } \\
& \left.\wedge \exists \operatorname{xrpc}: / / \text { uri } \in D\left(v_{x}\right)\right\} .
\end{aligned}
$$

This definition is also used by the next two algorithms to filter $d$-points. 
TABLE 4

Query Decomposition and Code Motion

\begin{tabular}{|c|}
\hline $\begin{array}{l}\qquad Q_{2}^{v} \text { : decomposed } Q_{2}^{n} \text { under pass-by-value } \\
\text { declare function fcn1() as node() } \\
\text { \{doc(“xrpc://A/students.xml”)/child::people/child::person }\} ;\end{array}$ \\
\hline 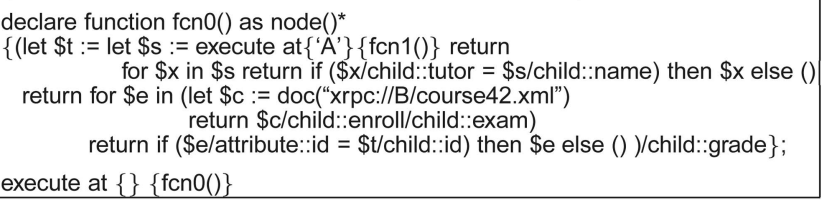 \\
\hline$Q_{2}^{f}:$ decomposed $Q_{2}^{n}$ under pass-by-fragment \\
\hline $\begin{array}{l}\text { declare function fcn } 1() \text { as node }()^{*} \\
\{\text { let } \$ \text { s := doc(“xrpc://A/students.xml")/child::people/child::person return } \\
\text { for } \$ x \text { in } \$ \text { s return if (\$x/child::tutor = \$s/child::name) then \$x else () \}; }\end{array}$ \\
\hline 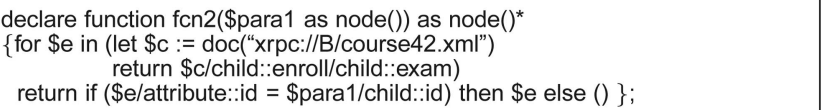 \\
\hline $\begin{array}{l}\text { declare function fcn } 0() \text { as node }()^{*} \\
\left\{\text { let } \$ t:=\text { execute at }\left\{{ }^{*} \mathrm{~A}\right\}\{\mathrm{fcn} 1()\}\right. \\
\left.\left.\text { return (execute at }\left\{{ }^{\prime} \mathrm{B} \text { ' }\right\}\{\mathrm{fcn} 2(\$ \mathrm{t})\}\right) / \text { child::grade }\right\} \\
\text { execute at }\{\}\{\mathrm{fcn} 0()\}\end{array}$ \\
\hline $\begin{array}{l}\text { Applying Distributed Code Motion in } Q_{2}^{f} \\
\text { declare function fcn2new(\$para2 as xs:::string } * \text { ) as node }()^{*} \\
\{\text { for } \$ \text { e in (let ... return ... ) return if (\$e/attribute::id }=\$ \text { para2) then } \$ \text { e else }()\} ;\end{array}$ \\
\hline $\begin{array}{l}\text { declare function fcn } 0() \text { as node }()^{*} \\
\left\{\text { let } \$ \mathrm{t}:=\text { execute at }\left\{\mathrm{A}^{\prime}\right\}\{\mathrm{fcn} 1()\} \text { return }\right. \\
\left.\left.\quad \text { let } \$ 1:=\$ \text { t return (execute at }\left\{\mathrm{B}^{\prime}\right\}\{\text { fcn } 2 \text { new }(\$ 1 / \text { child::id })\}\right) / \text { child:: grade }\right\}\end{array}$ \\
\hline
\end{tabular}

Example 4.2. In Fig. 2, the two subtrees rooted at $v_{5}$ and $v_{25}$ correspond to two different equivalent classes

$$
D\left(v_{5}\right)=\left\{\operatorname{xrpc}: / / \mathrm{A} / \text { students.xml }:: v_{9}\right\}
$$

and $D\left(v_{25}\right)=\left\{\operatorname{xrpc}: / / \mathrm{B} /\right.$ course $\left.42 . \mathrm{xml}:: v_{27}\right\}$. However, $v_{25}$ is not a valid insertion point. The vertices in $I^{\prime}(G)$ (colored dark gray) are $v_{6}$ (the highest non Var vertex in the subtree rooted at $v_{5}$ ) and the root $v_{1}$. Thus, $I^{\prime}(G)=\left\{v_{1}, v_{6}\right\}$.

Normalization. Rewriting algorithms that operate on the XCORE level are vulnerable to syntactic variation. In the case of our decomposition strategy, an important vulnerability comes from the behavior of the strategy to ship subgraphs consisting of parse-edges only. That is, varrefedges are not pushed, but rather become parameters to the function. The syntactic freedom one has in XQUERY of defining subexpressions, e.g., inline or via a variable reference to a previous let-binding, therefore affects our strategy. For this purpose, as part of XCORE normalization, we reorder let-bindings, moving them as deep into the query as possible. More specifically, let-bindings are moved to just above the lowest common ancestor vertex (defined in terms of parse-edges) of all vertices that reference its variable. The query $Q_{2}^{c}$ (Table 3) can be normalized to $Q_{2}^{n}$ (Table 3), which can thus be rewritten as $Q_{2}^{v}$ in Table 4.

The main achievement of normalization in the above case is to relate the call to doc(“../course42.xml") through parseedges (directly calling $\$ c$ in $Q_{2}^{n}$ ), instead of varref edges (referencing $\$ c$ in $Q_{2}^{c}$ ), with its use in the /child::enroll/ child::exam XPATH steps. However, these being part of a ForExpr with the /grade step on top, causes insertion Condition 2 to prohibit pushing it. In the next section on pass-by-fragment, however, we will see that normalization was not in vain, and the query can be decomposed into $Q_{2}^{f}$ (Table 4).
Distributed code motion. The let-normalization phase has the effect of pushing expressions that depend on the same documents downwards, potentially below an interesting insertion point (which makes them be executed remotely). However, it can happen that some of the expressions initially found below an interesting insertion point can in fact better be moved above it (to be executed locally). In particular, it is safe to assume that expressions that solely depend on a parameter of a function, can better be evaluated on the caller side. Moving a subexpression out of a function can be done by passing that subexpression as an additional parameter to the function. With pass-by-value passing, such a rewrite may not always be safe, however if only $d$-points are moved, the technique is semantically safe. Analogous to well-known compiler technique of moving invariant statements out of the loop (and its use in parallel processing [17]) we call this technique distributed code motion.

Example 4.3. Consider the function fcn2() in Table 4, we may observe that the expression \$para1/child::id only depends on the function parameter \$para1. Shipping full person nodes \$para1 from peer $A$ to $B$, only to extract the string value of its id child at $B$, may waste bandwidth, especially if person carries much more data than just an id. Instead, it would be better to extract the string value of id at peer A and only ship the strings. This optimization can be realized by adding a new parameter \$para2 to the function, and substituting \$para1/child::id in the body with it. In the function $\mathrm{fcn} 0($ ) that calls fcn2new(), we save the original function parameter $\$$ t in a new let -binding $\$$, and pass $\$ 1$ instead of $\$$ t. The additional function parameter is passed as $\$ 1 /$ child::id. Finally, the affected function parameter \$para1 is no longer used, so we remove it, arriving at the result as the code motion part in Table 4.

\section{By-Fragment Decomposition}

The node copying done by pass-by-value is the main source of semantic differences. This, in turn, leads to serious restrictions in the way the decomposition strategy can push expressions remotely. For this reason, we extend the passby-value message passing semantics into a new pass-byfragment message passing semantics that better preserves structural relationships of XML nodes. The basic idea is to avoid serializing the same nodes twice, by grouping all node-valued data in the message in a preamble element fragments. In principle, each node parameter is serialized below a separate fragment child element. However, if a sent node is a descendant of another one, it is not serialized twice, as we can reuse the XML fragment of the other node. We also ensure that the XML fragments are sorted in original document order, which means that ancestor/ descendant relationships in the same message, as well as node identity and document order, are preserved.

Later in the message, where XQUERY sequences are serialized (inside sequence tags), we just provide references to the nodes that were previously serialized in the fragments. In particular, element tags, which are used to contain as a child the fully serialized copy of a node, now just carry two numeric attributes, fragid and nodeid. In order to keep XRPC an interoperable protocol that is easy to implement for XQUERY engines and the XRPC WRAPPER 
TABLE 5

By-Value versus By-Fragment Messages

Excerpt of pass-by-value message for earlier $(\$ b c, \$ a b c)$

$\langle$ call $\rangle\langle$ sequence $\rangle\langle$ element $\rangle\langle b\rangle\langle c\rangle\langle\langle b\rangle\langle$ /element $\rangle\langle/$ sequence $\rangle$

$\langle$ sequence $\rangle\langle$ element $\rangle\langle a\rangle\langle b\rangle\langle c /\rangle\langle/ b\rangle\langle/ a\rangle\langle$ lelement $\rangle\langle/$ sequence $\rangle\langle/$ call $\rangle \mid$

Excerpt of pass-by-fragment message for earlier(\$bc,\$abc)

$\langle$ env:Envelope ...

$\langle$ env:Body $\rangle$

〈request>

〈fragments $\rangle\langle$ fragment $\rangle\langle a\rangle\langle b\rangle\langle c /\rangle\langle/ b\rangle\langle/ a\rangle\langle/$ fragment $\rangle\langle/$ fragments $\rangle$

$\langle$ call $\rangle\langle$ sequence $\rangle\langle$ element fragid=" 1 " nodeid="2" $\rangle\langle/$ sequence $\rangle$

$\langle/$ request $\rangle$

sequence $\rangle\langle$ element fragid="1" nodeid="1" $\rangle\langle/$ /sequence $\rangle\langle/$ call $\rangle$

$\langle/$ env:Body $\rangle\langle/$ env:Envelope

[38], node referencing is also expressible in XQUERY. Supposing \$msg is the root of the message, with \$fragid and $\$$ nodeid numbers, we can identify the referenced nodes as follows: ${ }^{4}$

\section{\$msg//fragment[\$fragid]/descendant::node()[\$nodeid]}

Example 5.1. Going back to $Q_{1}$ in Table 1, the lower part of Table 5 shows the XRPC request message sent for the call execute at $\{$ "example.org"\} \{earlier (\$bc, \$abc)\} from the discussion of Problem 3. Recall that the node \$bc with value $\langle b\rangle\langle c /\rangle\langle/ b\rangle$ is contained in the \$abc fragment $\langle a\rangle\langle b\rangle\langle c /\rangle\langle/ b\rangle\langle/ a\rangle$. The lower part of the figure shows an excerpt of the message as produced for pass-by-fragment. Here, both node parameters \$bc and \$abc are represented in element nodes with fragid and nodeid attributes. The XQUERY engine handling the call will use these attributes to evaluate:

\$bc := \$msg:fragment[1]/descendant::node()[2],
\$abc:= \$msg:fragment[1]/descendant::node()[1]

such that earlier $(\$ b c, \$ a b c)$ correctly returns \$abc, because \$abc «\$bc, just like on the peer that invoked this function. The upper part, with the changed part of the old pass-by-value message (element call), shows that node parameters were previously repeatedly serialized, causing node order and identity relationships between parameters to be lost.

We made a conscious choice not to rely on ID/IDREF for referencing nodes, since this would require adding ID attributes to the XML data in the fragments. As XRPC is designed to respect and conserve XML SCHEMA type information, this would cause the XRPC message to no longer respect user-defined schemas.

By-fragment insertion conditions. Given a d-graph $G$ and a subgraph $G_{r_{s}}$ of $G$ rooted at $r_{s}$, under the pass-by-fragment semantics, vertex $r_{s}$ is in the set $I(G)$ of valid decomposition points, iff $r_{s}$ satisfies all of the following conditions:

1.

$$
\begin{gathered}
\nexists n \in V(G): n . r u l e \in\{\operatorname{RevAxis}, \operatorname{Hor} A x i s\} \\
\wedge\left(\operatorname{useResult}\left(n, r_{s}\right) \vee \operatorname{useParam}\left(r_{s}, n\right)\right) ;
\end{gathered}
$$

4. Note that descendant::node() does not return attribute nodes. We use the nodeid of its parent and include the name of the attribute in an attribute element, so it can be found back with an additional attribute step.
2.

$$
\begin{aligned}
& \nexists n \in V(G): \text { n.rule } \in\{\text { NodeCmp, NodeSetExpr }\} \\
& \wedge\left(\left(r_{s} . \text { rule } \in\{\text { NodeCmp, NodeSetExpr }\}\right.\right. \\
& \left.\wedge n \notin V\left(G_{r_{s}}\right) \wedge \operatorname{hasMatchingDoc}\left(n, r_{s}\right)\right) \\
& \vee\left(\left(\text { useResult }\left(n, r_{s}\right) \vee \operatorname{useParam}\left(r_{s}, n\right)\right)\right. \\
& \wedge \text { hasMatchingDoc }(n, n)))
\end{aligned}
$$

3.

$$
\begin{aligned}
& \nexists n \in V(G), \exists m \in V(G): n . r u l e=\text { AxisStep } \wedge m . r u l e \\
& \quad \in\{\text { ForExpr, ExprSeq, NodeSetExpr }\} \\
& \wedge\left(( \text { useResult } ( n , m ) \wedge m \sim r _ { s } ) \vee \left(\text { useResult }\left(n, r_{s}\right)\right.\right. \\
& \left.\left.\wedge m \in V\left(G_{r_{s}}\right)\right) \vee\left(m \in V(G) \backslash V\left(G_{r_{s}}\right) \wedge r_{s} \stackrel{p}{\sim} n \stackrel{v}{\sim} m\right)\right) \\
& \wedge \text { hasMatchingDoc }(m, m) ;
\end{aligned}
$$

4.

$$
\begin{aligned}
& \nexists n \in V(G): \text { n.rule }=\text { FunCall } \wedge \text { n.val } \in\{\text { fn }: \operatorname{root}(), \\
& \text { fn }: \operatorname{id}(), \text { fn }: \operatorname{idref}(), \text { fn }: \operatorname{lang}()\} \wedge\left(\text { useResult }\left(n, r_{s}\right)\right. \\
& \left.\vee \text { useParam }\left(r_{s}, n\right)\right) .
\end{aligned}
$$

Thus, with the pass-by-fragment semantics, we modify the pass-by-value decomposition conditions listed in Section 4 by restricting the prohibitions to decompose a node $r_{s}$ formulated in Conditions 2 and 3 to only those $r_{s}$, for which the predicate hasMatchingDoc() holds:

$$
\begin{aligned}
& \text { hasMatchingDoc }\left(v_{1}, v_{2}\right) \Leftrightarrow \forall \operatorname{uri}_{l}:: v_{i} \in D\left(v_{1}\right), \\
& \quad \exists \text { uri }_{r}: v_{j} \in D\left(v_{2}\right): v_{i} \neq v_{j} \wedge\left(\text { uri }_{l}=\text { uri }_{r} \vee \text { uri }_{l}=* \vee \text { uri }_{r}=*\right) .
\end{aligned}
$$

By stating that the given expressions depend on two different applications of fn:doc() with the same URI (taking into account computed URIs as wildcards), this predicate precisely isolates the problem of creating result sequences with remote nodes from multiple calls to the same document.

The ForExpr is a special form of combining the results of multiple calls. A remote call nested in a for-loop, which depends on the same remote document, is treated as a single call, since bulk RPC ensures that all iterations of the remote call nested in the for-loop, are handled in a single message exchange (where pass-by-fragment now ensures proper conservation of node relationships). Finally, we remove from Condition 3 the restrictions that arbitrary ordering (OrderExpr) cannot be used and that all pushed AxisSteps should be of the nonoverlapping kind (parent, preceding-sibling, following-sibling, self, child, and attribute), as the pass-by-fragment message passing is able to properly conserve sequence order and the ancestor/descendant relationships between transported nodes. As the remaining problems with mixed-call sequences are related to dealing with multiple network message exchanges in the same query, this problem can not be solved inside the message passing semantics alone and is beyond our current scope. The restrictions to avoid horizontal and reverse XPATH steps on remote nodes (Condition 1) and on using built-in functions (Condition 4) will be addressed in the next section: 
TABLE 6

Pass-By-Projection Messages

\begin{tabular}{|l|}
\hline \multicolumn{1}{|c|}{ Excerpt of request message for makenodes ()} \\
\hline$\langle$ request $\rangle$ \\
$\langle$ projection-paths $\rangle$ \\
$\langle$ used-path $/\rangle$ \\
$\langle$ returned-path $\rangle$ parent::a $\langle/$ xrpc:returned-path $\rangle$ \\
$\langle$ projection-paths $\rangle$ \\
$\langle$ fragments $/\rangle$ \\
$\ldots$ \\
\hline \multicolumn{1}{|c|}{ Excerpt of response message for makenodes ()} \\
\hline$\langle$ env:Envelope $\ldots\rangle$ \\
$\langle$ env:Body $\rangle$ \\
$\langle$ response $\rangle$ \\
$\langle$ fragments $\rangle\langle$ fragment $\rangle\langle a\rangle\langle b\rangle\langle c /\rangle\langle/ b\rangle\langle/ a\rangle\langle/$ fragment $\rangle\langle/$ fragments $\rangle$ \\
$\langle$ call $\rangle\langle$ sequence $\rangle\langle$ element fragid=“'1" nodeid=“2”" $\rangle\langle/$ sequence $\rangle\langle/$ call $\rangle$ \\
$\langle/$ request $\rangle$ \\
$\langle$ lenv:Body $\rangle$ \\
$\langle/$ env:Envelope $\rangle$ \\
\hline
\end{tabular}

Example 5.2. Consider Fig. 2, as the constraint hasMatchingDoc() in Condition 3 does not hold, all vertices in the graph are identified as valid decomposition points under the pass-by-fragment semantics. However, most vertices will be filtered out by the definition of interesting decomposition points, which leads to $I^{\prime}(G)=\left\{v_{1}, v_{2}, v_{4}, v_{6}, v_{22}, v_{24}\right\}$.

\section{By-Projection Decomposition}

The basic idea of using XML projection [21] is, for a given XQUERY query $Q$ and an XML document $\mathcal{D}$, to extract a minimal part of data $\mathcal{D}^{\prime}$ needed to execute $Q$ such that $Q(\mathcal{D})=Q\left(\mathcal{D}^{\prime}\right)$. The projection technique conducts a compiletime path analysis on $Q$, to derive a set of simple path expressions that over-estimate the nodes that $Q$ touches. These simple paths are referred to as projection paths. Here, a projection path is an XML path that starts from the document root, containing forward navigation but not predicates (e.g., $\operatorname{doc}(\$ u r i) / a / b / @ i d)$. Projection paths consist of returned paths and used paths. Returned paths describe the nodes that are returned by the expression. Used paths indicate the nodes necessary to answer the query but are never returned as results (e.g., predicates). Based on the projected paths $\mathcal{P}$ of query $Q$ from path analysis, a loading algorithm is applied to $\mathcal{P}$ and an XML document (from a file or a stream) $\mathcal{D}$. A projected XML document (or stream) $\mathcal{D}^{\prime}$ is then generated, which contains all used and returned nodes plus the descendants of the returned nodes, and is queried with $Q$.

There are three reasons why projecting XML is extremely interesting for distributed XML processing: 1) until now, when sending nodes, we had to serialize all descendantswhich potentially contain huge subtrees that may remain untouched on the other side. This amounts to wasted network bandwidth as well as serialization and shredding effort. 2) if documents are projected into lean skeletons that only contain the relevant portions, it becomes feasible to serialize XML fragments from some lowest common ancestor on, possibly even the document root. Even with pass-byfragment, the execution of reverse/horizontal XPATH axes on remote nodes is impossible. By extending projecting XML with support for reverse and horizontal axes, however, we
TABLE 7

Grammar Rule Extension of Projectionpath (Bold)

get a tool to precisely identify the lowest common ancestor of an XML document that needs to be included to allow correct remote execution of those axes. 3) The projection technique can even be applied to support the built-in functions fn:root(), fn:id(), fn:idref() and fn:lang(), i.e., by taking the lowest common ancestor of those, if a path contains one of these functions. For these reasons, we further refine the passby-fragment message passing semantics into a so-called pass-by-projection semantics. XML projection can be used in both directions: to project the parameters in a request message, and to project the function's result sequence before shipping back the response.

Insertion conditions. Pass-by-projection removes the byfragment insertion conditions (in Section 5) 1 and 4, such that only 2 and 3, i.e., the application of node comparison, node set operators and axis steps on top of multiple calls to fn:doc() with the same URI, remains illegal.

Message extension: projection paths. We introduce an optional element as a subelement of a request tag: projectionpaths, which in turn has zero or more child elements returned-path and used-path. In the new pass-by-projection semantics, the absence or presence of this element determines whether the response message should be in the original pass-by-value or the new pass-by-projection format.

Example 6.1. To illustrate projected XRPC messages, the upper part of Table 6 shows part of the request message for the call from $Q_{1}$ (discussed in Problem 4):

let $\$ \mathrm{bc}:=$ execute at $\{$ "example.org" $\}$ makenodes()\}

since the projection path analysis detects that $\$ b c$ will subsequently be used as context node by a parent step: $\$ a b c:=\$ b c /$ parent::a, the request message specifies parent::a as a returned path. Therefore, the response message contains the full fragment $\langle a\rangle\langle b\rangle\langle c /\rangle\langle/ b\rangle\langle/ a\rangle$ to which \$abc then gets correctly bound.

\subsection{Extending Projected XML}

We extend the path grammar rules [21] and path annotations, to handle full-fledged XQUERY involving reverse/ horizontal XPATH steps and built-in functions. The extended grammar rule for ProjectionPath is given in Table 7.

We denote path annotations in projected XML as:

$$
\text { Env }\left(v_{i}\right) \vdash \operatorname{Expr} \Rightarrow \text { Paths using UPaths. }
$$

The notation $\operatorname{Env}\left(v_{i}\right)$ is used to identify the path annotation environment at a certain vertex $v_{i}$ in the XQUERY $d$-graph.

Path annotations are constructed bottom up by path analysis rules that derive the set of used (UPaths) and returned (Paths) paths for each XCORE expression in terms of used and 


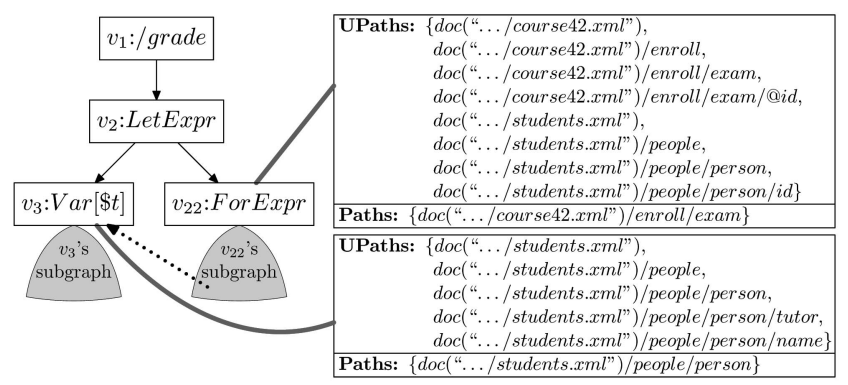

Fig. 4. Path annotation example.

returned paths of its subexpressions. Therefore, we extend the notation of the vertices and use $v_{i}$.UPaths and $v_{i}$.Paths to refer to the path sets, with which the vertex $v_{i}$ is annotated.

Example 6.2. Assume that the subgraph $G_{v_{22}}$ rooted at $v_{22}$ in Fig. 2 is identified to be evaluated remotely. The subgraph $G_{v_{22}}$ has one parameter, $\$ t$, via the VarRef edge $\left(v_{36}, v_{3}\right)$. We show the path annotations of $v_{3}$ and $v_{22}$ in Fig. 4. Comparing the returned path of $v_{3}$ with all projection paths of $v_{22}$ and, $v_{1}$, we know that $v_{3}$ is only used in the subgraph rooted at $v_{22}$ (i.e., it is not returned by $v_{22}$ ), and that only the id child elements of the person elements are used. Thus, only those elements will be projected and serialized in the request message for $v_{22}$. For reasons of space, document URIs are abbreviated, and the annotations of $v_{1}$ and $v_{2}$ are omitted, which in reality also have such annotations.

The basic path analysis rules have been discussed in [21], such as literal values, sequences, for and let expressions, and $\mathrm{XPATH}$ steps, etc. Our extension to include reverse and horizontal XPATH steps brings no changes for the path analysis rules, but must be supported by the loading algorithm, which is described in Section 6.2. We complement the rules for built-in functions, which apart from the unsolved cases mentioned under Problem 5 in Section 2 (fn:root(), fn:id(), fn:idref(), and fn:lang()) also includes fn:doc(). The description of the basic projection technique assumes a single document. As in distributed query processing there are always multiple documents, our paths always start with fn:doc(URI).

Path analysis rules. We provide one rule for fn:doc() with a constant parameter and another for computed URIs:

$$
\begin{aligned}
& \frac{()}{\operatorname{Env}\left(v_{i}\right) \vdash \text { fn:doc }\left(\text { Literal }_{1}\right) \Rightarrow \text { fn:doc }\left(\text { Literal }_{1}:: v_{i}\right) \text { using } \emptyset} \quad\left(\text { DOC }_{1}\right) \\
& \underline{\operatorname{Env}\left(v_{j}\right) \vdash \operatorname{Expr}_{j} \Rightarrow \text { Path }_{j} \text { using UPaths }} s_{j} \\
& E n v\left(v_{i}\right) \vdash \text { fn:doc }\left(E x p r_{j}\right) \Rightarrow \text { fn: } \operatorname{doc}\left(*:: v_{i}\right) \text { using } \\
& \text { Paths }_{j} \cup \text { UPaths } s_{j} \cup \text { Paths }_{j} \text { /descendant::text() }
\end{aligned}
$$

As mentioned in Section 4 , in the definition of $D\left(v_{x}\right),{ }^{5}$ we use a wildcard URI $*$ if the document name is an expression. All paths start with doc(uri:: $\left.v_{i}\right)$, thus, they identify both document URI and the vertex $v_{i}$ where it is loaded. This notation facilitates the identification of situations where the same URI is loaded twice (the function hasMatchingDoc()).

5. We use the $\operatorname{doc}(.$.$) prefixes of the returned paths annotations on v$ as a more precise form of the $D(v)$ property. Documents that were only used but not returned will also be part of the original $D(v)$, but these will not cause semantic problems.
A similar rule can be formulated for XML element construction, producing a return path $\operatorname{doc}\left(v_{i}: v_{i}\right)$ with an artificial unique URI. Also note that XQUERY implicitly converts the actually values of function parameters, thus, in all rules in this section, we add a descendant::text() step to each returned path of a parameter. The rule for fn:root() is:

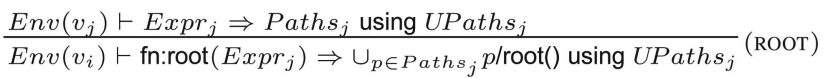

The built-in function fn:root() with a single parameter is treated in the path annotations much like XPATH axis steps, where the parameter has become the path prefix. In this path notation, functions remain easily recognisable by the parentheses. The rules for the built-in functions fn:id()/ fn:idref(), are highly similar (only fn:id() provided):

$$
\begin{aligned}
& E n v\left(v_{j}\right) \vdash \text { Expr }_{j} \Rightarrow \text { Paths }_{j} \text { using UPaths } \\
& \text { Env }\left(v_{k}\right) \vdash \text { Expr }_{k} \Rightarrow \text { Paths }_{k} \text { using UPaths } \\
& k
\end{aligned}
$$

The first parameter of $f n: i d()$ is ignored by the annotations, as it contains string values, and the annotation framework only allows for the estimation of node sets. This has the consequence that our loading algorithm will conserve all elements with an ID/IDREF attribute. Finally, the rule for fn:lang() is:

$$
\begin{aligned}
& \operatorname{Env}\left(v_{j}\right) \vdash \operatorname{Expr}_{j} \Rightarrow \text { Paths }_{j} \text { using UPaths } \text { U }_{j} \\
& \operatorname{Env}\left(v_{k}\right) \vdash \operatorname{Expr}_{k} \Rightarrow \text { Paths }_{k} \text { using UPaths } s_{k} \\
& \operatorname{Env}\left(v_{i}\right) \vdash \text { fn:lang }\left(\text { Expr }_{j}, \text { Expr }_{k}\right) \Rightarrow \emptyset \text { using Paths } s_{j} \cup \text { UPath }_{j} \cup \\
& \text { Path } s_{j} \text { /descendant::text() } \cup \text { Paths } s_{k} \cup \text { UPath } s_{k} \cup \\
& \text { Path } s_{k} \text { /ancestor::* } \cup \text { Path } s_{k} \text { /ancestor-or-self::*/attribute::xml:lang }
\end{aligned}
$$

The built-in function fn:lang() tests whether the language of its node parameter $E \mathrm{xpr}_{k}$, as specified by xml:lang attributes, is the same as (or is a sublanguage of) the language specified by its string parameter Expr. The language of Expr $r_{k}$ is determined by the value of the XPATH expression: (ancestor-or-self::*/attribute::xml:lang)[last()]. All paths are propagated as used paths, as this function returns a boolean value.

\subsection{Runtime XML Projection}

The extensions we made to XML projection, namely support for reverse/horizontal XPATH axes and fn:root(), fn:id(), fn:idref(), and fn:lang(), could not be trivially integrated in the loading algorithm of [21]. However, in this case we are not really looking for a loading algorithm that efficiently reads (shreds) an XML file into a projected representation. Rather, the documents are already present (and indexed) in the XQUERY engine, and runtime message projection is a serialization task. Therefore, we propose a new runtime approach for projection, targeted at serialization, rather than at shredding. Whereas the original loading algorithm starts at the document root, and evaluates absolute used and returned paths, our runtime projection algorithm starts in a runtime state, that is, with a real, materialized context sequence (e.g., the parameter values that are about to be serialized in a SOAP message), and executes only relative paths on them. Because the node sequence bound at runtime to a function parameter is only a subset of the node set characterized by 
its compile-time path annotation (e.g., its contents may well have been reduced by applying a selection predicate), this runtime projection technique can be much more precise than the original projection algorithm. As a final consideration, the projected XRPC messages trade projection effort for network bandwidth, which especially in WAN scenarios plays in the advantage of projection.

For these reasons, our runtime approach for projection simply relies on the normal XPATH evaluation capabilities of the XQUERY engine for fully evaluating all used and returned path annotations one-by-one (and uniting them with union()). Doing so, it produces a used node set $U$ and a returned node set $R$. These two sets are the input for the runtime projection algorithm listed in Algorithm 1.

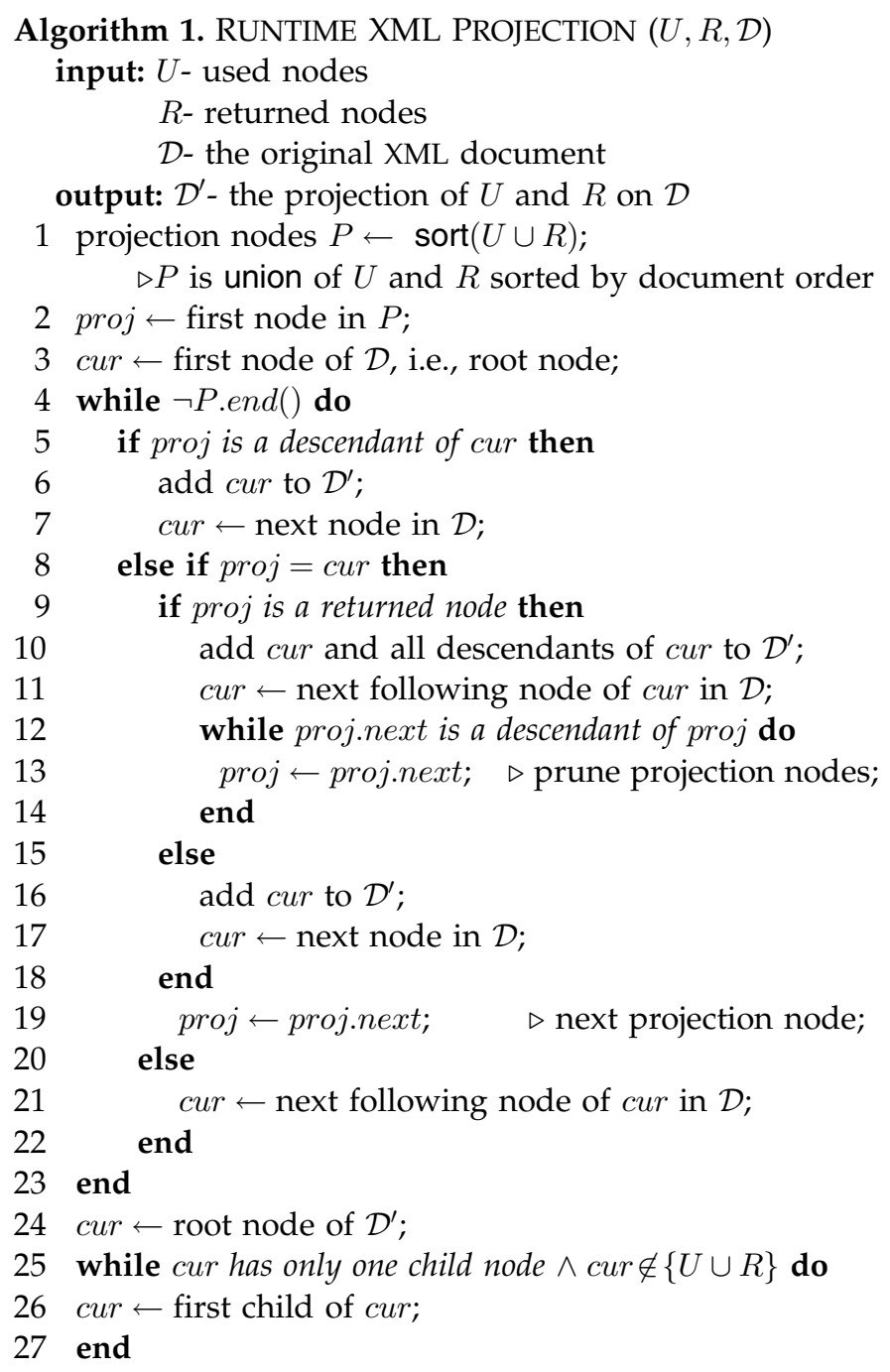

The runtime projection algorithm. This algorithm identifies all projection nodes in the XML tree representation of the original document, by traversing the tree top-down depth-first. During traversal, if the current node cur of the XML document is an ancestor of the current projection node proj (line 5), cur is added to output $\mathcal{D}^{\prime}$ and moved to the next node in document order. If a proj is found (line 8), proj is added to $\mathcal{D}^{\prime}$; if this proj is a returned node, all its descendants are also appended. Then, cur is moved to its next following node in the document. Otherwise, if the current projection node proj is not a descendant of cur, the subtree of cur can be skipped (line 21). Though this

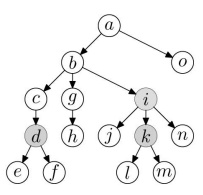

(a)

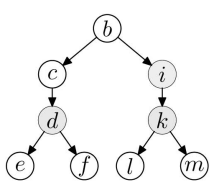

(b)
Fig. 5. Runtime XML projection example (a) original tree $\mathcal{D}(b)$ projected tree $\mathcal{D}^{\prime}$.

algorithm is formulated on an abstract level that is independent of the particular XML storage scheme used in an XQUERY engine, it is safe to assume that skipping a subtree is fast (either $O(1)$ or $O(\log (|\mathcal{D}|)))$. At the end of the algorithm (lines 24-27), postprocessing is performed to remove unnecessary nodes, as we are only interested in the lowest common ancestor of all input nodes in the projected document $\mathcal{D}^{\prime}$.

Example 6.3. Consider an XML document $\mathcal{D}$ in Fig. 5a. Assume that the used node set $U$ is $\{i\}$, and the returned node set $R$ is $\{d, k\}$. Fig. $5 \mathrm{~b}$ shows the projected document $\mathcal{D}^{\prime}$ of applying Algorithm 1 on $U, R$ and $\mathcal{D}$.

The algorithm starts with $P \leftarrow\{d, i, k\}$, proj $\leftarrow d$ and cur $\leftarrow a$. We traverse the tree using cur from $a$ to $d$. Nodes $a, b$, and $c$ are added to $\mathcal{D}^{\prime}$, since they are ancestors of the current context node $d$. Nodes $d, e$, and $f$ are also added to $\mathcal{D}^{\prime}$, as $d$ is a returned node. Then, cur is advanced to $g$ ( $d$ 's next following node). Because the next context node $i$ is not in the subtree of $g$, the subtree is skipped by advancing cur to $i$. Recall that $i$ is a used node, thus only $i$ is added to $\mathcal{D}^{\prime}$. The last context node is $k$. Our current document node cur traverses from $i$ to $j$, and then to $k$, where we can add nodes $k, l$, and $m$ to $\mathcal{D}^{\prime}$. The traversal can be terminated, because there is no more context nodes to process. However, the intermediate result $\mathcal{D}^{\prime}$ contains all common ancestors of $\{d, i, k\}$. The postprocessing removes node $a$ from $\mathcal{D}^{\prime}$, which produces the final projected document $\mathcal{D}^{\prime}$ as shown in Fig. $5 b$.

Relative projection paths. At compile time, the XQUERY compiler builds a query graph ( $d$-graph) with root $v_{\text {root }}$, normalizes it followed by decomposition and code motion. For each inserted XRPCExpr $v_{x r p c}$, and for each XRPCParam parameter vertex $v_{\text {param }}$, it then extracts the relative paths:

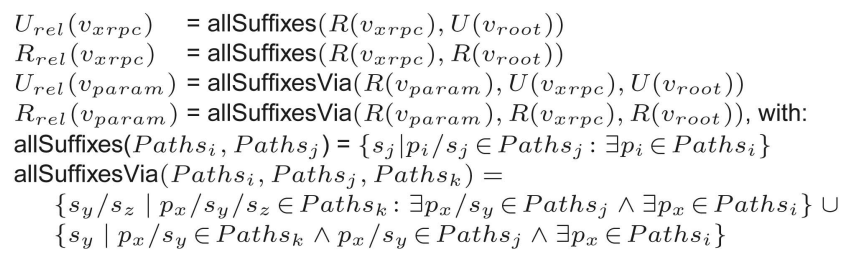

At runtime, $\cup_{\forall v_{\text {param }}} U_{\text {rel }}\left(v_{\text {param }}\right)$ and $\cup_{\forall v_{\text {param }}} R_{\text {rel }}\left(v_{\text {param }}\right)$ are used to project the parameters in the outgoing XRPC request message. $U_{r e l}\left(v_{x r p c}\right)$ and $R_{r e l}\left(v_{x r p c}\right)$ are passed in the projection-paths element such that a remote peer can appropriately apply these paths to project the response message. When computing the relative used and returned paths for $v_{\text {param }}$, we need to take into account that (parts of) $v_{\text {param }}$ could be returned by $v_{x r p c}$, and thus will be used by vertices depending on $v_{x r p c}$. Hence, in allSuffixesVia(), we not only 
find the relative paths that $v_{x r p c}$ will apply on $v_{\text {param }}$, but also the relative paths that $v_{\text {root }}$ will apply on $v_{\text {param }}$. In both auxiliary functions, we use a dot "." to denote a self step, if two paths from different sets equal to each other. If, for instance, a returned path of $v_{\text {root }}$ equals to a returned path of $v_{x r p c}$, there will be a dot "." in $R_{\text {rel }}\left(v_{x r p c}\right)$ indicating that the XML subtrees returned by $v_{x r p c}$ should be serialized.

Projecting a document using Algorithm 1 requires precalculated used and returned node sets. These sets are simply computed using the XPATH evaluation infrastructure of the underlying XQUERY engine, by feeding the intermediate result $\$ \mathbf{c t} \mathbf{x}_{\text {param }}$ corresponding to $v_{\text {param }}$ as context sequence into all suffix paths $s_{i} \in U_{\text {rel }}\left(v_{\text {param }}\right)$ (respectively, $R_{\text {rel }}\left(v_{\text {param }}\right)$ ):

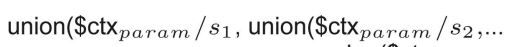

$$
\text { union } \left.\left.\left(\$ \operatorname{ctx}_{\text {param }} / s_{n-1}, \$ \operatorname{ctx}_{\text {param }} / s_{n}\right) \ldots\right)\right)
$$

Paths $\$ c t x / p a t h_{i} / \operatorname{root}() / p^{2} h_{j}$ with function fn:root() are executed as root(\$ctx) $/ p a t h_{j}$. Similarly, $\$ c t x / p a t h_{i} / \mathrm{id}() / p a t h_{j}$ is executed as $\operatorname{root}(\$ \operatorname{ctx}) / /$ attribute ()$::\left(a_{1}|..| a_{n}\right) / . . / p a t h_{j}$, where $a_{1}, . ., a_{n}$ are all ID attributes ${ }^{6}$ (resp., IDREF in case of idref()).

The request handler on the remote side uses the same method to evaluate the suffix paths $U_{\text {rel }}\left(v_{x r p c}\right)$ and $R_{\text {rel }}\left(v_{x r p c}\right)$ using the result sequence of the function as $\$ \operatorname{ctx}_{x r p c}$ during serialization of the response message.

Interoperability. We have devised a way to support pass-by-projection in the XRPC WRAPPER by substituting the projection algorithm with a variant that serializes the lowest common ancestor of the used and returned node sets. Since document projection is not expressible in XQUERY (not even with the TRANSFORM feature of XQUF), this is as far as a pure XQUERY engine can get. We contemplate the possibility to let the XRPC WRAPPER echo the SOAP response message it generates to a stream, and implement a streaming version of our projection algorithm (that first gets a stream of used and returned nodes, and then the to be projected fragments) inside the XRPC WRAPPER java program. In case of XML data with a userdefined XML SCHEMA, the default projection algorithm is likely to throw away mandatory elements and attributes. For this reason, the runtime projection algorithm should be made schema-aware. A simple solution is to ensure that only elements with a minoccurs declaration of zero (i.e., optional elements) are removed. One can also envision more advanced variants that further reduce the size of a typed XML document.

\section{DECOMPOSITION OF XQUF QUERIES}

Since the introduction of the W3C XQUF [7] specification, which has been well received and adopted by various XQUERY engines (e.g., [9], [11], [16], [23], [26], [27], [36]), XQUERY is no longer a read-only query language. We now show how we can leverage such update-capable XQUERY engines to automatically rewrite purely local updates into queries that may push some computations to remote peers. We recall that the general processing model of XQUF is that

6. Note that these $a_{i}$ should be determined at runtime by the XRPC projection algorithm. The impossibility to express selection of all ID/IDREF attributes in XQUERY, and thus in the XRPC WRAPPER, forces us to still avoid shipping expressions where the result of $v_{x r p c}$ is used as input to id()/idref(). first the read-only part of a query is executed that defines which nodes are going to be updated, and how. This first phase results in a pending update list (PUL). In the second phase all update actions in this list are executed. Therefore, the first phase of XQUF execution is identical to a read-only query, and can in principle be distributed in the same way as described in the previous sections. However, systems implementing the XQUF typically only allow to update persistently stored documents, e.g., updating documents on an HTTP URI is not allowed. In this section, we first explain the restrictions the XQUF imposes on XRPC query distribution. Then, we extend the semantics of XQUF to allow updates on documents opened with fn:doc() using xrpc://P/ $\mathcal{D}$ URIs (in short: remote documents) and also support the fn:put()XQUF built-in function to write entire new documents to such URIs. This extended semantics again creates a possible trade-off between data shipping versus function shipping, namely retrieving and updating a local copy of a remote document followed by an fn:put() versus executing an XQUF updating function over XRPC. We introduce the necessary constraints to our query distribution techniques that guarantee semantic equivalence for such queries.

\subsection{Distributing Normal XQUF Queries}

XQUF has extended the XQUERY language with four kinds of updating expressions: UpdExpr $=\{$ InsertExpr, DeleteExpr, RenameExpr, ReplaceExpr\} (Table 2). An XCORE query containing at least one UpdExpr is an updatingXCOREquery (in short: updating query). Each UpdExpr has a TargetExpr that identifies the target nodes to be updated, and (except for DeleteExpr) each has an ExprSingle that computes the new values. For simplicity, we refer to those ExprSingle as SrcExpr, although XQUF uses different names. The functionality of the first three kinds of expressions is self explanatory. With ReplaceExpr, one can replace the target node with a new sequence of nodes ("replace node"), or replace the value of the target node ("replace value"). The expressions RenameExpr and "replace value" only modify some properties of the target node without changing its node identity.

XQUF also defines a transform expression (TransformExpr) that creates (and possibly modifies) copies of existing XML nodes. Each node created by a TransformExpr has a new node identity. The result of a TransformExpr is an XDM (XQuery Data Model) instance that may include both new nodes created by the TransformExpr and existing nodes. TransformExpr has special semantics: it is not an updating expression, as it does not modify any existing nodes. Hence, an XCORE query that merely contains UpdExpr as subexpressions of a TransformExpr is not an updating query.

In our XCORE rewriting framework, all three algorithms use a by-value based semantics, which means that target nodes may not stem from an XRPC function result, or from a function parameter (if the updating expression occurs inside an XRPC function body). Hence, we enforce that all UpdExprs, denoted $V_{u}$, must be executed on the same peer that opened the document using $f n: \operatorname{doc}()$. This, in turn, enforces that all expressions $V_{a_{i}}$ (except TransformExpr), which depend on a $v_{u_{i}} \in V_{u}$, must be executed on the local peer. This is because $V_{a_{i}}$ could only parse-depend on a $v_{u_{i}}$, as updating expressions are not allowed in a variable 
binding. Decomposing an expression in $V_{a_{i}}$ would cause the $v_{u_{i}}$ to be executed on a remote peer. To correctly identify the target nodes of an UpdExpr, all expressions $V_{t_{i}}$ that produce target nodes for a $v_{u_{i}}$, must also be executed on the local peer. When decomposing an updating query, the vertices $V_{u}, V_{t_{i}}$, and $V_{a_{i}}$ in the query's $d$-graph are never valid decomposition points, regardless of the parameter passing semantics used by the decomposition algorithm. The following XQUF insertion conditions should be added to the insertion conditions of each decomposition algorithm.

XQUF insertion conditions. Given a $d$-graph $G$ and a subgraph $G_{r_{s}}$ of $\mathrm{G}$ rooted at vertex $r_{s}$, under any semantics, $r_{s}$ is in the set $I(G)$ of valid decomposition points, iff $r_{s}$ also satisfies all of the following conditions:

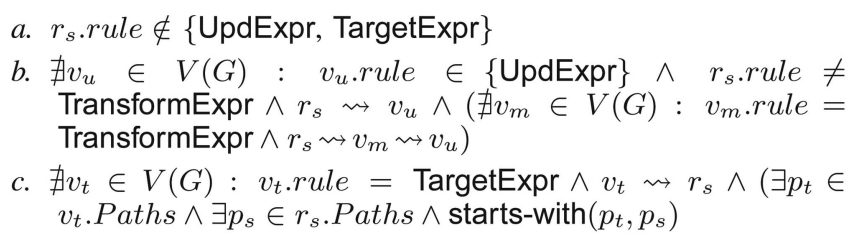

Condition $a$ avoids decomposing any UpdExpr and Target-Expr. Condition $b$ states that if $r_{s}$ is not a TransformExpr, $r_{s}$ may not depend on an UpdExpr, unless the UpdExpr is a subexpression of a TransformExpr, on which $r_{s}$ depends. Condition $c$ states that $r_{s}$ may not be decomposed, if $r_{s}$ produces target nodes of an UpdExpr. We say $r_{s}$ produces target nodes, iff a returned path $p_{s}$ of $r_{s}$ is a prefix of a returned path $p_{t}$ of $v_{t}$, i.e., nodes returned by $r_{s}$ include target nodes. Note that condition $b$ allows a TransformExpr to be decomposed by all three decomposition algorithms, as it always makes (deep) copies of its source nodes. If a TransformExpr is executed on peer $\mathcal{P}, \mathcal{P}$ becomes the "local peer" for all new nodes created by this TransformExpr. With Condition $a$, we prevent UpdExprs in the modify clause of a TransformExpr from being separated from the TransformExpr (i.e., executed on another peer than $\mathcal{P})$. Thus, the UpdExprs in the modify clause will also be executed on $\mathcal{P}$, which is the local peer of their target nodes. This confirms the XQUF semantics that UpdExprs may only be applied to local nodes. In the remainder of this section, we continue our discussion on processing UpdExprs that are not subexpressions of a TransformExpr.

\subsection{Updating XCore Queries on Remote Documents}

We now extend the semantics of XQUF to allow updates on remote documents (i.e., documents identified by an xrpc:// URI scheme). We first provide a semantics for such updates in normal nondistributed execution (i.e., data shipping): the read-only part of the query is evaluated first, retrieving (a copy of) all accessed remote documents to the local peer, which results in a PUL. Then, the standard XQUF function upd:applyUpdates() is executed to carry through all update actions in the PUL. This could modify (some of) the local copies of the remote documents. Finally, as an additional step, for each affected remote document, an fn:put() is executed by passing the document's original URI and its new contents, effectively replacing the existing document on the remote peer with the modified one. This semantics does not apply to XCORE queries only containing transform expressions, as they are read-only queries. Thus, no additional fn:put() is executed to overwrite the existing documents.
Formal semantics. Let $Q_{u}$ denote an XCORE query containing at least one UpdExpr on a remote document and $G_{u}$ its $d$-graph. $D_{u}\left(Q_{u}\right)$ denotes the set of affected documents that may be updated by $Q_{u}$ :

$$
\begin{gathered}
D_{u}\left(Q_{u}\right)=\left\{(\text { uri },) \mid \exists v_{u}, v_{y}, v_{z} \in V\left(G_{u}\right):\left\{v_{y}, v_{z}\right\} \in E\left(G_{u}\right) \wedge\right. \\
\text { uri }=v_{z} . \text { val } \wedge \text { node }=\operatorname{doc}(\text { uri }) \wedge v_{y} . \text { rule }=\text { FunApp } \wedge \\
v_{y} . \text { val }=\text { "doc" } \wedge v_{z} . \text { rule }=\text { Literal } \wedge v_{u} . \text { rule }=\text { TargetExpr } \wedge \\
\text { uri } \left.=v_{z} . \text { val } \wedge \exists p \in v_{u} . \text { Paths } \wedge \text { starts-with }(p, \text { uri })\right\}
\end{gathered}
$$

$D_{u}^{r}\left(Q_{u}\right)$ is a subset of $D_{u}\left(Q_{u}\right)$, which contains the affected remote documents:

$$
\forall d_{i}^{r} \in D_{u}^{r}\left(Q_{u}\right): \operatorname{starts}-\operatorname{with}\left(d_{i}^{r} . u r i, " x r p c: / / "\right) .
$$

The auxiliary functions host() and path() extract, from an XRPC URI "xrpc://P/D $/ \mathcal{D}$ ", the peer identifier $\mathcal{P}$ and the document name $\mathcal{D}$, respectively. Each query operates in a database state $\left(d b^{p}\right)$, which includes the documents and their contents persistently stored in the XML database on $p$. The dynEnv.docValue from [10] corresponds to $d b^{p}$ used here.

The formal semantics of distributed updates is ${ }^{7}$ :

$$
\begin{aligned}
& \forall d_{x}^{r} \in D_{u}^{r}\left(Q_{u}\right): \text { fn:doc }\left(d_{x}^{r} . u r i\right) \Rightarrow D_{u}^{r^{\prime}}\left(Q_{u}\right) \\
& d b^{p_{0}}, D_{u}^{r^{\prime}}\left(Q_{u}\right) \vdash Q_{u} \Rightarrow \Delta ; \\
& d b^{p_{0}}, D_{u}^{r^{\prime}}\left(Q_{u}\right) \vdash \text { upd:applyUpdates }(\Delta) \Rightarrow d b^{\prime p_{0}}, D_{u}^{r^{\prime \prime}}\left(Q_{u}\right) ; \\
\left(R^{u}\right) \forall d_{x}^{r^{\prime \prime}} \in D_{u}^{r^{\prime \prime}}\left(Q_{u}\right): \text { fn:put }\left(d_{x}^{r^{\prime \prime}} . \operatorname{node}, d_{x}^{r^{\prime \prime}} . u r i\right) \Rightarrow(), d b^{\text {host }\left(d_{x}^{r^{\prime \prime}} \cdot \text { uri }\right)} ; & d b^{p_{0}} \vdash Q_{u} \Rightarrow(), d b^{p_{0}}
\end{aligned}
$$

The rule $R^{v}$ states that the execution of an updating query $Q_{u}$ at the local peer $p_{0}$ in the database state $d b^{p_{0}}$ starts with retrieving the remote documents $D_{u}^{r}\left(Q_{u}\right)$, which could potentially be affected by $Q_{u}$, to $p_{0}{ }^{8}$ This yields a set of local copies $D_{u}^{r^{\prime}}\left(Q_{u}\right)$ of $D_{u}^{r}\left(Q_{u}\right)$. Note that this step does not change $d b^{p_{0}}$, as the documents in $D_{u}^{r^{\prime}}\left(Q_{u}\right)$ are transient documents. Then, $Q_{u}$ is executed in $d b^{p_{0}}$ with the additional documents $D_{u}^{r^{\prime}}\left(Q_{u}\right)$, which first yields a PUL $\Delta$. Subsequently, upd:applyUpdates() is executed to apply all update primitives in $\Delta$ to the affected documents. Updates in $\Delta$ that should be applied on remote documents $D_{u}^{r}\left(Q_{u}\right)$ are applied on their local copies $D_{u}^{r^{\prime}}\left(Q_{u}\right)$ instead. This step produces a new current database state $d b^{\prime p_{0}}$, which could differ from $d b^{p_{0}}$ (if $\Delta$ contains updates on really local documents), and a set of changed local copies $D_{u}^{r^{\prime \prime}}\left(Q_{u}\right)$. Finally, an additional step is executed, which calls fn:put() to store each $d_{i}^{r^{\prime \prime}} \in D_{u}^{r^{\prime \prime}}\left(Q_{u}\right)$ on its hosting peer and overwrite the existing $d_{i}^{r} \in D_{u}^{r}\left(Q_{u}\right)$. This step also creates a new current remote database state $d b^{\text {host }\left(d_{x}^{\prime \prime} \text {.uri }\right)}$ on each hosting peer. As the rule $R^{u}$ only applies $\Delta$ at the end of query execution, updates are not visible for the same query, which confirms the XQUF semantics. Hence, if $\Delta$ only contains updates on a single document, this rule already provides atomic updates.

Isolation levels. Note that the-potentially multiple-fn:put("xrpc://..") together with potential updates on some local documents constitute a distributed updating query. Depending on the semantics desired by the user, this distributed updating query could be run in a certain consistency level, which has been discussed in detail in

7. We use the ":" sign to suggest an order in the evaluation of the premises.

8. As explained in Section 4, computed URIs and invocations of fn:collection() are represented by $*$. During the runtime, when the actually value of the wildcard symbols are available, more URIs might be added to the set $D_{u}^{r}\left(Q_{u}\right)$ on the fly. 


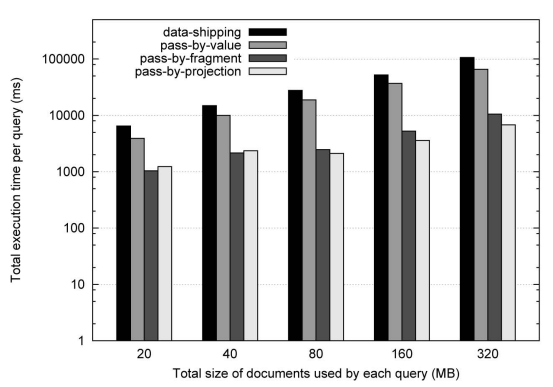

Fig. 6. Execution time.

of roughly 94 percent, comparing with data-shipping; this is proportional to the decreasing in bandwidth usage, which is approximately 96 percent. Even on small documents $(20 \mathrm{MB})$, the proposed techniques are preferred over the data-shipping methods.

Fig. 7 shows the execution time breakdown of all four queries on documents of $320 \mathrm{MB}$ in total. The execution time is divided into five parts: shred is the time to receive a document from the remote peer and shred it in to the XML database; local exec is the execution time of the query at local peer, including query parsing, module loading, etc; (de)serialize is the time spent on generating/shredding XML messages and extracting parameter/result values from the messages; remote exec is the time to execute the called functions on remote peers; and network is the time spent on sending/receiving XML messages. From Fig. 7, the following observations can be made: 1) in the data-shipping-only query and the by-value-decomposed query, data shredding is the main bottleneck, either because the whole document is shipped (data-shipping), or an XML node might be shredded multiple times (by-value). Especially in the datashipping query, more than 99 percent of the total execution time is spent on getting the documents from remote peers and shredding them; 2) when pass-by-fragment and passby-projection semantics are used, the total execution time is significantly reduced (about 84 94 percent, comparing with data-shipping and pass-by-value). This is easily explained as these techniques reduce the amount of data exchanged to be less than 10 percent of the original document sizes. Even with the overhead introduced by remote execution (i.e., "(de)serialize" + "remote exec"), passby-fragment or pass-by-projection are preferred over the data-shipping method. 3) pass-by-projection performs even better than pass-by-fragment (about 35 percent improvement), which is again explained by the reduced bandwidth usage, as shown in Fig. 8.

Bandwidth usage. Fig. 8 shows the bandwidth used by each benchmark query on different set of documents, i.e., the total size of XML documents plus total size of XML messages transferred among peers, in its $y$-axis. The $x$-axis is the total size of the XML documents used by each query. The pure datashipping XQUERY query (the left most bar) costs the largest bandwidth usage, as both documents used by the query have to be shipped. By-value decomposition can push the XPATH step doc(“xrpc://peer1/xmk_nn_MB.xml”)/.../child::person to be evaluated on peer1, which reduces the amount of data sent from peer1 to the local peer. However, the second document "xmk_nn_MB.auctions.xml" still has to be sent fully. The byfragment passing semantics allows to push predicates to both

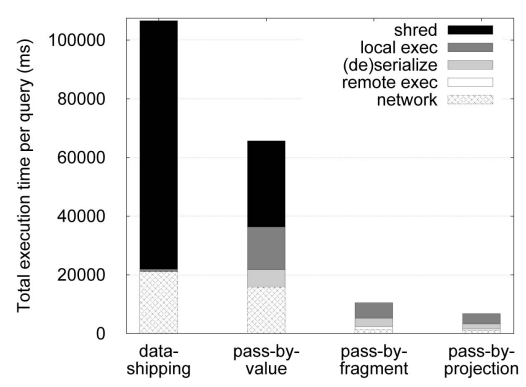

Fig. 7. Time breakdown (320 MB data).

peers, achieving a distributed semijoin plan. Also, it strongly reduces message size by avoiding duplicating the same XML node multiple times. Pass-by-projection further brings down message sizes due to reduced response message size. For example, when sending the result of remote execution of the second for-loop, the response message will only contain annotation nodes with their author child nodes. When applied on pass-by-fragment, code motion has larger effect in reducing message size than it is applied on pass-byprojection. This is because in pass-by-fragment, complete person nodes (i.e., including all their descendants) are serialized, while in pass-by-fragment with code motion, only the values of the id attributes are serialized. In pass-byprojection, however, the message size has already minimized the data to be sent, i.e., only person nodes and their id attributes, hence, the effect of applying code motion here is negligible. In general, we observe good scalability of pass-byfragment and pass-by-projection in bandwidth usage.

Runtime projection precision. Our new runtime projection technique combines intermediate query results with runtime execution or relative XPATH paths. Due to selections (by, e.g., predicates and value comparisons), the runtime projection node sets obtained may be much smaller than suggested by compile-time projection paths, used in [21]. We used our by-projection benchmark query to compare runtime projection with compile-time projection, on various sizes of the XMark document "xmk_nn_MB.xml". In this experiment, the compile-time technique projects all person elements and their age, while our runtime projection technique will only project those person elements that have an age descendant larger than 45. Fig. 9 shows runtime projection to be five times more precise in terms of the size of projected document. In the case of this experiment, the investment in runtime XPATH evaluation pays off due to the more precise results, as shown in Fig. 10.

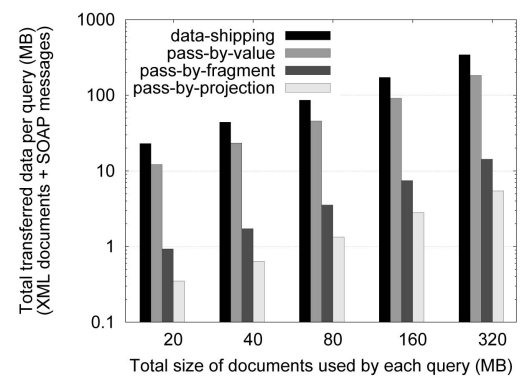

Fig. 8. Bandwidth usage. 


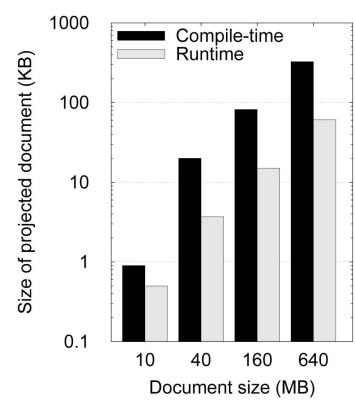

Fig. 9. Selected nodes.

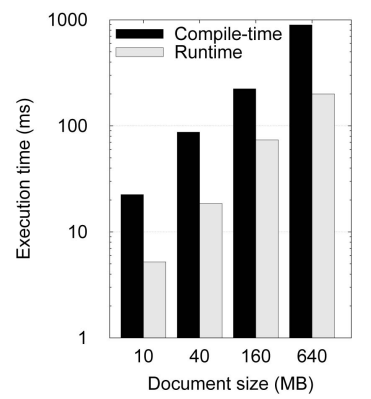

Fig. 10. Execution time (ms).

\subsection{XQUF Queries}

For the updating XCORE queries, we have conducted two groups of experiments to compare performance of updating remote documents with or without XRPC. The first group corresponds to the generic strategy discussed in Section 7 where a remote document is first retrieved to the local machine (with fn:doc()), then the updates are applied on the local copy of the remote document, and finally the updated document is written to the remote peer using fn:put(). We call queries in this group "GUPqueries" (i.e., Get-UpdatePut). In the second group, called "XRPCqueries", updates are applied directly on the original document at the remote peer with XRPC using so-called updating functions as specified by the XQUF:

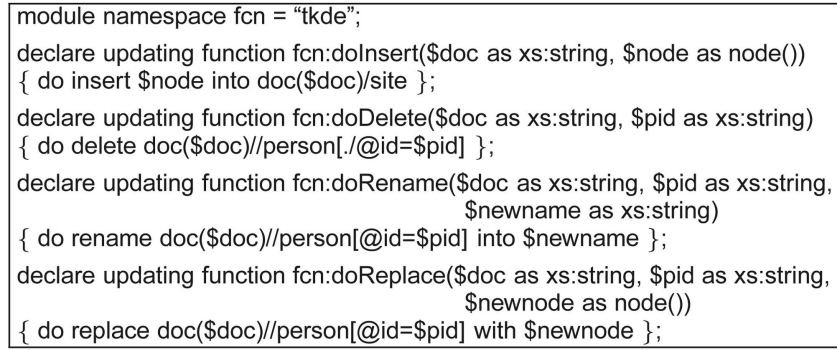

We tested all four kinds of updates, keeping the granularity of the updates constant, affecting 100 person nodes. For example, the insert query in XRPC looks as follows:

import module namespace fcn="tkde" at "http://example.org/tkde.xq";

fcn:dolnsert("xrpc://p2/xmark200mb.xml”, doc(“Persons100.xml")/persons)

All updating queries were applied on XMark documents of $200,400,600,800$, and 1,000 MB, respectively. The data set is stored on one peer, which acts as the remote peer. The total execution time of all queries are shown in Fig. 11.

For all four kinds of update queries, XRPC is significantly faster than GUP. The relatively small performance

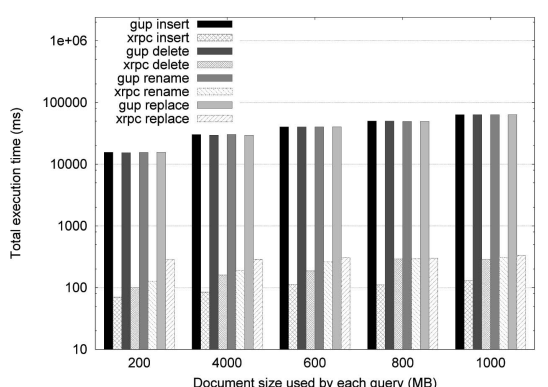

Fig. 11. Updates execution time.

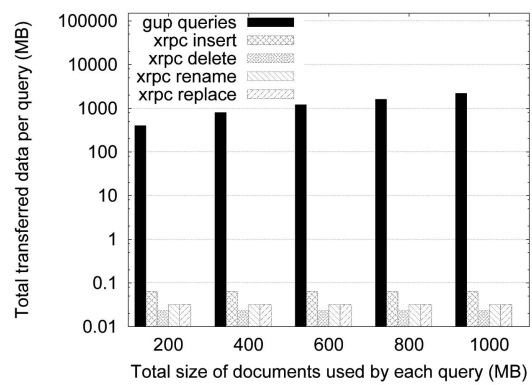

Fig. 12. Updates bandwidth usage.

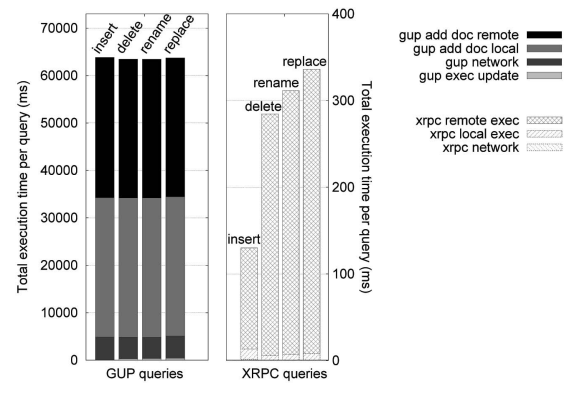

Fig. 13. Updates time breakdown (1,000 MB).

differences between different kinds of updates reflects the MonetDB/XQuery implementation of the XQUF. We can conclude that with increasing document sizes, the absolute benefits of XRPC grow linearly, which is caused by the additional full serialization, network copy, and shredding for the "Get" phase, followed by full serialization and network copy steps in the "Put" phase, performed by the GUP approach. As the amount of updates is small, the total bandwidth usage of all GUP queries are approximately twice the documents size, as shown in Fig. 12, whereas the XRPC query only sends the function parameters and results (tens of KB). In Fig. 13, the bars at the left-hand-side show the time breakdown of GUP queries, while the bars at the right-hand-side show the time breakdown of XRPC queries; all were run on a 1 GB document. From Fig. 13, it can be seen that the GUP queries spend a large amount of time on adding the document to the local and remote database (shown as "gup add doc remote" and "gup add doc local"). They also spend a significant amount of time on exchanging the document between the local peer and the remote peer (shown as "gup network"). However, the times spent on actually applying the updates (shown as "gup exec update") are only a very small portion of the total execution times. On the other hand, for the XRPC queries, the only dominant factor in the total execution 
time is the time spent on applying the updates (shown as "xrpc remote exec"), while the times spent on processing the request and response messages (i.e., serialize, send and deserialize) are negligible.

We finally recall that in all experiments (including the read-only ones) we used a local area network (LAN); but in a WAN environment, where much lower network performance is common, the benefits of our query decomposition techniques will be larger, as we showed by their strongly reduced network bandwidth use.

\section{Related Work}

There are three main areas that are related to our proposal in this paper: distributed query processing, query decomposition and XML projection.

Much previous work in distributed query processing is surveyed in [19], [37] and parts of the book [25]. In distributed XML query processing, DXQ [13] depends on distributed query plans, in terms of the internal Galax execution algebra, generated by the Galax optimizer. In this respect, XRPC differs with its focus on interoperability, as it acts as a pure XQUERY rewriter (not making any assumptions on the system internals of the participating peers). Galax Yoo-Hoo [24] accesses web services using SOAP RPC as the communication protocol, which lacks proper support for XML elements and sequences; a problem addressed by XRPC using a specific literal SOAP message encoding. Active XML (AXML) [1] is a declarative framework that harnesses web services for data integration in a P2P architecture. Like $\mathrm{XRPC}$, it also uses a (document/literal encoding) SOAP protocol to represent XML subtree values. However, the focus in AXML has been in adaptive call materialization strategies, not on automatic query decomposition and the semantic challenges this brings in XQUERY, such as distributed node identity. XQueryD [28] supports function shipping in XQUERY like XRPC, but it does not define an open network protocol.

Decomposing queries to address multiple data sources is by now a well-studied problem in relational [34] and objectoriented [15], [20] DBMS. Many of these ideas and methods can be applied to XQUERY, yet we have shown here that issues of efficiently managing distributed node identity and document order add interesting challenges. [30], [31] discuss the decomposition of unstructured query languages only on a semistructured database (a rooted, labeled graph). In XML databases, previous approaches require structural information about peers for supervising decomposition [33]. Other works, e.g., [6], [8], [32] only focus on a restricted subset of XQUERY queries. This paper is based on our previous work on decomposing read-only XQuery queries [40]. As one of the main goals of our work is to support fullfledged XQuery, in this paper, we have made our framework more complete by extending [40] with analysis on how updating queries can be decomposed. Some preliminary experiments have been done to show the performance improvements that could be achieved by executing updates directly on remote documents at the remote peers, instead of first retrieving a remote document to the local peer, then applying updates on the local copy of the document, and finally put the updated document back to the remote peer.
XML projection [21] drastically reduces the size of the data model representation using compile-time query characterization. Bressan et al. [5] introduces a precise XML pruning technique for a subset of XQUERY FLWOR expressions, based on the a priori knowledge of a data guide for underlying XML data. However, it does not handle XPATH predicates, backward axes, and XQUERYlike languages. A type-based XML projection technique [2] is studied to improve current solutions with comparable or higher precision and less pruning overhead, as well as supporting backward XPATH axes. However, a DTD is required. koch et al. [18] discusses runtime XML projection techniques. Based on the static compilation of runtime lookup-tables and a runtime-automaton from projection paths and a DTD, they can filter the input XML document efficiently using string matching algorithms. This technique, however, still lacks support for reverse XPATH axes and XQUERY built-in functions.

\section{Conclusion}

We have described a framework for distributed execution of full-fledged XQUERY including XQUF, focusing on the issue of providing equivalent query decompositions, in the face of semantic differences when (parts of) nodes are shipped across the network in XML messages. We first carefully characterized the problems that may occur regarding node identity and structural XPATH relationships in such a distributed setting. Then, we proposed a series of techniques such as pass-by-fragment and the use of a novel runtime XML projection method for serializing XML messages, that remove virtually all semantic problems and strongly improve performance, as shown by experiments on the open-source MonetDB/XQuery XDBMS (monetdb.cwi.nl). We also discussed the semantics of updating both local and remote documents using XQUF expressions, and additional constraints that should be added to the proposed techniques to guarantee semantic equivalence for such queries. Our main future work is an issue left out-of-scope here: deciding on distributed query placement after decomposition. In this area, we also contemplate using runtime methods to improve optimization quality.

\section{REFERENCES}

[1] S. Abiteboul, O. Benjelloun, B. Cautis, I. Manolescu, T. Milo, and N. Preda, "Lazy Query Evaluation for Active XML," Proc. ACM SIGMOD, 2004.

[2] V. Benzaken et al., "Type-Based XML Projection," Proc. Int'l Conf. Very Large Data Bases (VLDB), 2006.

[3] S. Boag et al., "XQuery 1.0: An XML Query Language," W3C Candidate Recommendation, June 2006.

[4] P. Boncz et al., "MonetDB/XQuery: A Fast XQuery Processor Powered by a Relational Engine," Proc. ACM SIGMOD, 2006.

[5] S. Bressan et al., "Accelerating Queries by Pruning XML Documents," Data Knowledge Eng., vol. 54, no. 2, pp. 211-240, 2005.

[6] P. Buneman et al., "Using Partial Evaluation in Distributed Query Evaluation," Proc. Int'l Conf. Very Large Data Bases (VLDB), 2006.

[7] D. Chamberlin et al., "XQuery Update Facility 1.0," W3C Candidate Recommendation, Aug. 2008.

[8] G. Cong et al., "Distributed Query Evaluation with Performance Guarantees," Proc. ACM SIGMOD, 2007.

[9] DataDirect XQuery, http://www.datadirect.com, 2010. 
[10] D. Draper et al., "XQuery 1.0 and XPath 2.0 Formal Semantics," W3C Recommendation, Jan. 2007.

[11] eXist, http://exist.sourceforge.org, 2009.

[12] M. Fernández et al., "XQuery 1.0 and XPath 2.0 Data Model (XDM)," W3C Candidate Recommendation, July 2006.

[13] M. Fernández et al., "Highly Distributed XQuery with DXQ," Proc. ACM SIGMOD, 2007.

[14] T. Grust et al., "XQuery on SQL Hosts," Proc. Int'l Conf. Very Large Data Bases (VLDB), 2004.

[15] V. Josifovski et al., "Query Decomposition for a Distributed Object-Oriented Mediator System," Distributed and Parallel Databases, vol. 11, no. 3, pp. 307-336, 2002.

[16] M. Kay., "SAXON The XSLT and XQuery Processor," http:// saxon.sourceforge.net, 2010.

[17] J. Knoop and B. Steffen, "Code Motion for Explicitly Parallel Programs," ACM SIGPLAN Notices, vol. 34, no. 8, pp. 13-24, 1999.

[18] C. Koch et al., "XML Prefiltering as a String Matching Problem," Proc. Int'l Conf. Data Eng. Conf. (ICDE), 2008.

[19] D. Kossmann, "The State of the Art in Distributed Query Processing," ACM Computing Surveys, vol. 32, no. 4, pp. 422-469, 2000.

[20] H. Kozankiewicz, K. Stencel, and K. Subieta, "Distributed Query Optimization in the Stack-Based Approach," High Performance Computing and Communications, Springer, 2005.

[21] A. Marian et al., "Projecting XML Documents," Proc. Int'l Conf. Very Large Data Bases (VLDB), 2003.

[22] N. Mitra and Y. Lafon, "SOAP Version 1.2 Part 0: Primer," second ed., W3C Recommendation, Apr. 2007.

[23] MonetDB/XQuery, http://monetdb.cwi.nl, 2010.

[24] N. Onose and J. Siméon, "XQuery at Your Web Service," Proc. Int'l World Wide Web Conf. (WWW), 2004.

[25] M.T. Özsu and P. Valduriez., Principles of Distributed Database Systems, second ed. Prentice-Hall, Inc., 1999.

[26] IBM DB2 pureXML, http://www-01.ibm.com/software/data/ $\mathrm{db} 2 / \mathrm{xml} /, 2010$.

[27] Qizx, http://www.qizx.com, 2010.

[28] C. Re et al., "Distributed XQuery," Proc. Workshop Information Integration on the Web (IIWeb), Sept. 2004.

[29] A. Schmidt et al., "XMark: A Benchmark for XML Data Management," Proc. Int'l Conf. Very Large Data Bases (VLDB), 2002.

[30] D. Suciu, "Query Decomposition and View Maintenance for Query Languages for Unstructured Data," Proc. Int'l Conf. Very Large Data Bases (VLDB), 1996.

[31] D. Suciu, "Distributed Query Evaluation on Semistructured Data," ACM Trans. Database System, vol. 27, no. 1, pp. 1-62, 2002.

[32] K. Tajima and Y. Fukui, "Answering XPath Queries Over Networks by Sending Minimal Views," Proc. Int'l Conf. Very Large Data Bases (VLDB), 2004.

[33] L.T.T. Thuy, D.D. Duong, V.C. Bhavsar, and H. Boley, "A BottomUp Strategy for Query Decomposition," Proc. Int'l Conf. Digital Information Management (ICDIM), 2006.

[34] E. Wong and K. Youssefi, "Decomposition-A Strategy for Query Processing," ACM Trans. Database System, vol. 1, no. 3, pp. 223-241, 1976.

[35] Web Services Atomic Transaction, ftp://www6.software.ibm. com/software/developer/library/WS-AtomicTransaction.pdf. Aug. 2005.

[36] XQilla, http://xqilla.sourceforge.net/, 2010.

[37] C. Yu and C. Chang., "Distributed Query Processing," ACM Computing Surveys, vol. 16, no. 4, pp. 399-433, 1984.

[38] Y. Zhang and P. Boncz, "XRPC: Interoperable and Efficient Distributed XQuery," Proc. Int'l Conf. Very Large Data Bases (VLDB), 2007.

[39] Y. Zhang and P. Boncz, "Distributed XQuery and Updates Processing with Heterogeneous XQuery Engines," Proc. ACM SIGMOD, 2008.

[40] Y. Zhang, N. Tang, and P. Boncz, "Efficient Distribution of FullFledged XQuery," Proc. Int'l Conf. Data Eng. (ICDE), 2009.

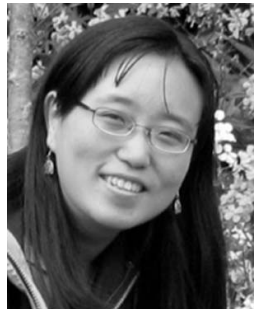

Ying Zhang received the MSc degree in computer networks from the Vrije Universiteit Amsterdam, where she has also worked for three years as an assistant in teaching various programming courses. Her master thesis is about optimization and adaptation of an epidemic protocol called Newscast in P2P file sharing applications. She is working toward the $\mathrm{PhD}$ degree in the database architecture research group (INS1) of CWI. For her PhD work, she has designed and implemented XRPC, a simple yet efficient extension of the full-fledged XQuery for distributed querying of XML data. Since 2005, she has been working on distributed XML database systems, which is part of the project MultimediaN, funded by the Dutch Government (BSIK).

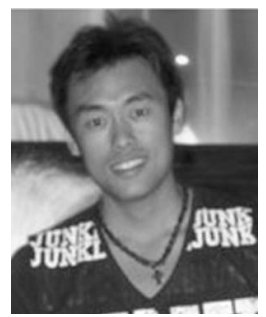

Nan Tang received the PhD degree in Department of Systems Engineering \& Engineering Management from The Chinese University of Hong Kong, Hong Kong, in 2007. Currently, he is a postdoctoral fellow in CWI (National Research Center from Mathematics and Computer Science of The Netherlands), Amsterdam, The Netherlands. His research interests include $\mathrm{XML}$ database management, scalable string matching for disk image, and updates for column-based database.

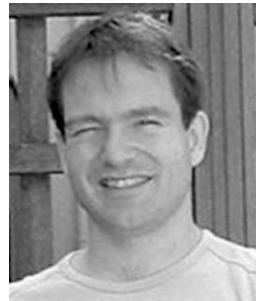

Peter Boncz is a tenured researcher at CWI, and coauthor of more than 50 scientific papers. His academic background is in core database architecture, with the architecture of the architecture-conscious columnar store MonetDB the main topic of his $\mathrm{PhD}$ thesis; which also led to being a corecipient of the 2009 VLDB 10 Year Award. In recent years, he has worked on (distributed) XML database systems as well as RDF. He also received the Dutch ICT Regie Award 2006 for bridging the gap between academia and commercial application in the CWI spin-off company Data Distilleries. In 2008, he founded a new CWI spin-off company called VectorWise, dedicated to state-of-the art business intelligence technology.

$\triangleright$ For more information on this or any other computing topic, please visit our Digital Library at www.computer.org/publications/dlib. 